\title{
First-principles thermal modeling of hybrid pseudocapacitors under galvanostatic cycling
}

\author{
Anna L. d'Entremont and Laurent Pilon* \\ University of California, Los Angeles, \\ Henry Samueli School of Engineering and Applied Science, \\ Mechanical and Aerospace Engineering Department, \\ 420 Westwood Plaza, \\ Los Angeles, CA 90095, USA
}

\begin{abstract}
This study presents a theoretical framework developed from first principles for predicting the spatiotemporal thermal behavior of hybrid pseudocapacitors under galvanostatic cycling. It accounts for irreversible and reversible heat generation in the electrolyte and in the electrodes due to Joule heating, electric double layer (EDL) formation, and redox reactions. Detailed numerical simulations were performed to investigate the different local heat generation rates and the temperature as functions of time and cycling current. Numerical predictions showed good qualitative agreement with the limited experimental data available in the literature. Such numerical simulations can be used to physically interpret experimental measurements. Indeed, the present results suggest that a distinctive endothermic peak observed in the experimental heat generation rate resulted from cation starvation in the electrolyte reducing the faradaic current density. In addition, heating due to EDL formation significantly affected the local temperature and must be accounted for. The thermal model and the present results will help define safe modes of operation and develop thermal management strategies for pseudocapacitors.
\end{abstract}

Keywords: pseudocapacitors; supercapacitors; electrochemical energy storage; Joule heating; heat generation; thermal management

${ }^{*}$ Corresponding author, Email: pilon@seas.ucla.edu, Tel: +1 (310) 206-5598, Fax: +1 (310) 2062302 


\section{Introduction}

Pseudocapacitors combine the chemical energy storage concept of batteries with the electrostatic energy storage concept of electric double layer capacitors (EDLCs) by storing electric charge both chemically via redox reactions and electrostatically within the electric double layer (EDL) [1-3]. They are classified, together with EDLCs, as electrochemical capacitors (ECs) or supercapacitors despite their use of faradaic charge storage because their electrical performance characteristics closely resemble those of EDLCs rather than those of batteries $[1,2]$. In particular, their cell potential varies continuously with their state of charge [4]. Hybrid pseudocapacitors are devices pairing a pseudocapacitive electrode with an EDLC-type electrode [4]. Pseudocapacitors typically feature larger power densities, cycle life, and cycle efficiencies than batteries as well as much larger energy densities than dielectric capacitors [5]. In particular, they provide larger capacitances and energy densities than EDLCs because they combine faradaic and electrostatic charge storage in order to accommodate more charge per unit electrode surface area than EDLCs $[2,4,6,7]$.

EDLCs, batteries, and pseudocapacitors all generate heat during operation due to various physical processes [8, 9]. Irreversible Joule heating arises from current flow through the electrodes and the electrolyte while reversible heat generation rates arise due to EDL formation in the electrolyte [10-12]. Exothermic or endothermic faradaic reactions provide additional heating or cooling. Heat generation in ECs can be problematic as excessive temperature rise accelerates aging $[8,10,13-16]$, increases self-discharge rates $[1,10,13-16]$, and increases faradaic reaction rates [17-19]. Unfortunately, few studies have investigated the thermal behavior of pseudocapacitors $[8,9,20]$. Detailed thermal modeling can be used to predict operating temperatures and to improve our understanding of the underlying causes of heat generation in order to develop thermal management strategies and mitigate negative thermal effects.

The present study aims to derive, from first principles, a rigorous thermal model to predict the spatiotemporal evolution of heat generation rates and temperature in hybrid pseudocapacitors. The model accounts for heating due to interfacial and transport processes 
within the electrodes and electrolyte and due to redox reactions. Such a thermal model and the associated simulation tool could aid in defining safe modes of operation and facilitate systematic development of thermal management strategies.

\section{Background}

\subsection{Experimentally observed thermal behavior of electrochemical capacitors}

Experimental studies of EDLCs have found that galvanostatic cycling under current $\pm I_{s}$ resulted in (i) an overall temperature rise from cycle to cycle attributed to irreversible heating and (ii) superimposed temperature oscillations at the cycle frequency attributed to reversible heating due to EDL formation and dissolution $[8-10,15,16,21,22]$. The irreversible heat generation rate was proportional to $I_{s}^{2}$. The reversible heat generation rate was exothermic during charging, endothermic during discharging, and proportional to $I_{s}[9,10,21]$.

Unfortunately, less experimental data investigating the thermal behavior of pseudocapacitors is available in the literature. Dandeville et al. $[9,23]$ measured the time-dependent heat generation rates during galvanostatic cycling of a hybrid pseudocapacitor consisting of an $\mathrm{MnO}_{2}$ positive electrode and an activated carbon negative electrode in $0.5 \mathrm{M}$ aqueous $\mathrm{K}_{2} \mathrm{SO}_{4}$ electrolyte. Here, $\mathrm{K}^{+}$ions from the electrolyte reacted reversibly with $\mathrm{MnO}_{2}$ so that the positive electrode charged by deintercalation of $\mathrm{K}^{+}$ions [24]. The authors also considered an EDLC consisting of two activated carbon electrodes identical to that used in the hybrid pseudocapacitor [9]. The heat generation rates in each carbon electrode, either in the EDLC or in the hybrid pseudocapacitor, were assumed to be identical at any given current. This enabled the authors to identify the reversible heat generation rates associated with each electrode in the hybrid pseudocapacitor. The total reversible heat generation rate associated with the pseudocapacitive $\mathrm{MnO}_{2}$ electrode was found to be (i) endothermic during charging by deintercalation of $\mathrm{K}^{+}$and (ii) exothermic during discharging by intercalation of $\mathrm{K}^{+}$ [9]. Its magnitude was also proportional to $I_{s}$ [9]. Note, however, that it was not possible to differentiate between faradaic and EDL contributions to the heat generation rate in the pseudocapacitive electrode. 


\subsection{Interfacial and transport phenomena in pseudocapacitors}

Recently, we presented a first-principles continuum model for the electrochemical interfacial and transport phenomena in hybrid pseudocapacitors under galvanostatic cycling [25]. It accounted simultaneously for ion intercalation in the pseudocapacitive electrode as well as EDL formation including steric effects in the electrolyte based on the generalized modified Poisson-Nernst-Planck (GMPNP) model [25]. Numerical simulations showed two asymptotic regimes: (i) a faradaic regime occurring for small imposed current density and slow charging when faradaic charge storage dominated and (ii) a capacitive regime occurring for large imposed currents and fast charging when EDL charge storage dominated. The results indicated that accounting simultaneously for EDL formation, faradaic reactions, and their effects on each other was essential for reproducing experimentally observed features of the cell potential [25]. The present study extends this model to consider the thermal effects.

\subsection{Thermal models of electrochemical capacitors}

Various thermal models have been proposed to predict the temperature within EDLCs by solving the heat diffusion equation in one [11, 12], two [14, 16, 22], or three dimensions $[21,26,27]$. In addition, several models have predicted the transient EDLC temperature while assuming uniform temperature throughout the device [28, 29] or by treating the device as multiple regions of uniform temperature connected by thermal resistances [13-15, 27]. The majority of thermal studies have treated EDLCs as "black boxes" with experimentally determined properties such as electrical resistance and capacitance [13-16, 26, 27]. The irreversible heat generation rate was typically modeled as either a constant input parameter $[14,16,21,26]$ or as uniform Joule heating (in $\mathrm{W}$ ) expressed as $I_{s}^{2} R$, where $R$ is the cell's experimentally measured electrical resistance $[10,13,15,27,29]$. Most thermal models have accounted only for irreversible heating and have neglected the reversible contribution [1416, 21, 26]. However, Schiffer et al. [10] developed an expression to estimate the reversible heat generation rate due to electrolyte entropy changes accompanying EDL formation. It was proportional to the imposed current density, as observed experimentally [10]. Other thermal models have modeled the reversible heating using this expression [13, 22, 29] or 
simply as the imposed current density multiplied by a fitting parameter [27, 28]. Finally, we recently derived a thermal model for EDLCs $[11,12]$ predicting the local, time-dependent irreversible and reversible heat generation rates from first principles based on the local ion concentrations and the local electric potential within the electrolyte.

There are fewer thermal models in the literature for pseudocapacitors. Srinivasan and Weidner [30] predicted the transient temperature evolution of a pseudocapacitor cell assuming (i) uniform temperature, (ii) uniform electrolyte concentration at all times, i.e., ignoring the EDL formation, and (iii) heat generation solely due to irreversible Joule heating [30]. Wang et al. [20] predicted the local temperature for a commercial hybrid pseudocapacitor under galvanostatic cycling by solving the 3D heat diffusion equation with heat generation. Here also, the authors neglected reversible heating and accounted only for irreversible Joule heating based on the experimentally measured resistance of the device [20]. The numerically predicted maximum temperature in the core of a commercial hybrid pseudocapacitor was considered to be in good agreement with experimental surface temperature measurements, although the model underpredicted the surface temperature by several degrees [20].

The present study aims to develop a local and time-dependent thermal model accounting for both irreversible and reversible heat generation rates arising from simultaneous and coupled EDL formation and faradaic reactions as well as from Joule heating in hybrid pseudocapacitors. The spatiotemporal model was based on first principles and rigorously accounted for the electrochemical interfacial and transport phenomena. Detailed numerical simulations of a hybrid pseudocapacitor with planar electrodes under galvanostatic cycling were performed to investigate its thermal behavior for various imposed current densities and cycle periods.

\section{Analysis}

\subsection{Schematic and assumptions}

Figure 1 illustrates the one-dimensional (1D) hybrid pseudocapacitor cell considered in the present study. It consisted of two current collectors supporting planar pseudocapacitive and carbon electrodes separated by a binary and asymmetric electrolyte with interelectrode 


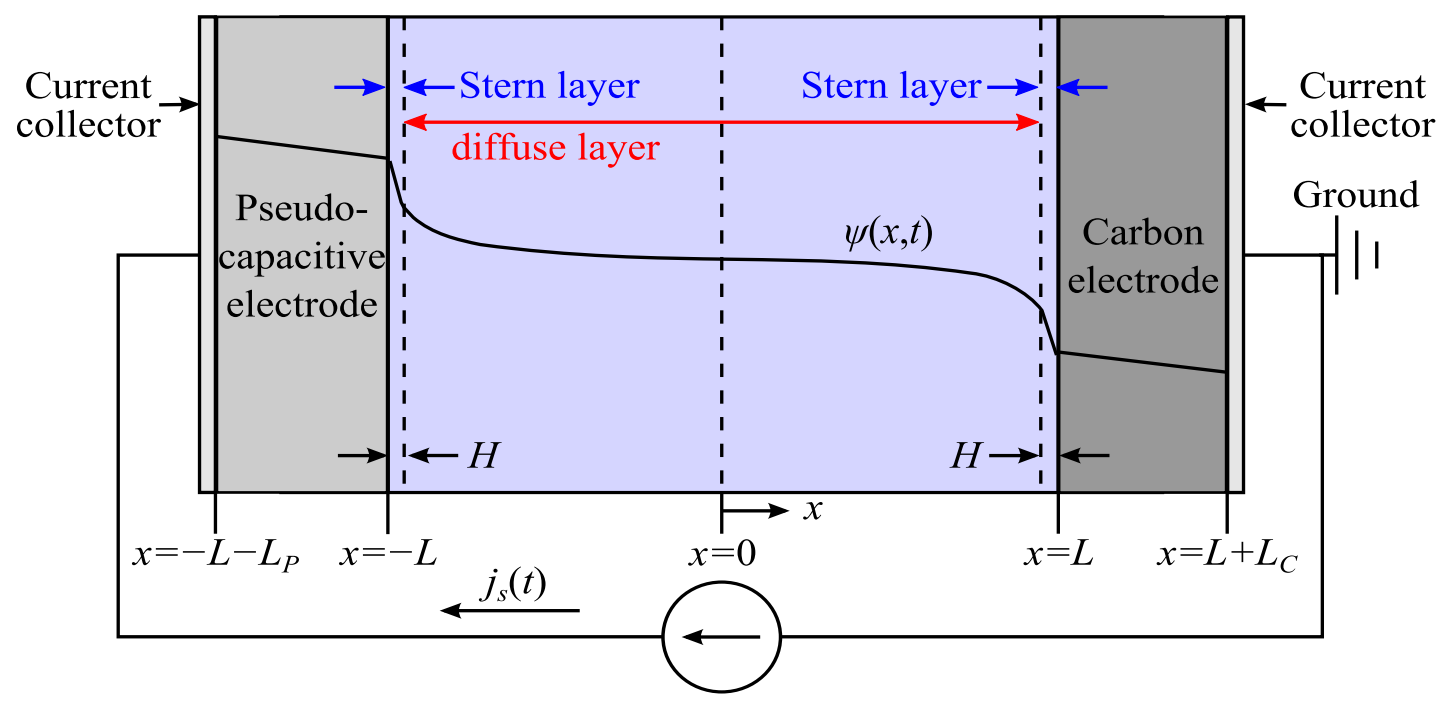

Figure 1: Illustration of the simulated hybrid pseudocapacitor with planar electrodes. The electrolyte was divided into the Stern and diffuse layers according to the Stern model $[17,18]$.

distance $2 L$. According to the Stern model $[17,18]$, the electrolyte was divided into two regions, namely the Stern and the diffuse layers. The compact Stern layer adjacent to the electrode surface contains no free charge $[17,18]$. By contrast, the diffuse layer contains ions moving under the competing influences of electrostatic forces, diffusion, and steric repulsion $[17,18,31]$. The Stern/diffuse layer interface marks the closest distance of approach for a solvated ion [19]. The pseudocapacitive electrode of thickness $L_{P}$ was located at $-L-L_{P} \leq$ $x \leq-L$ and consisted of a transition metal oxide $\left(\mathrm{M}_{p} \mathrm{O}_{q}\right)$ reacting chemically with lithium cations according to the following reversible redox reaction

$$
m \mathrm{Li}^{+}+\mathrm{M}_{p} \mathrm{O}_{q}+m \mathrm{e}^{-} \rightleftharpoons \mathrm{Li}_{m} \mathrm{M}_{p} \mathrm{O}_{q}
$$

where $m$ is the number of $\mathrm{Li}^{+}$ions intercalated per molecule of $\mathrm{M}_{p} \mathrm{O}_{q}$. The heterogeneous reaction occurring at the pseudocapacitive electrode/electrolyte interface transferred $\mathrm{Li}^{+}$ ions from the electrolyte into the $\mathrm{M}_{p} \mathrm{O}_{q}$ matrix [17-19]. Subsequently, the intercalated $\mathrm{Li}^{+}$ migrated farther into the pseudocapacitive electrode, accompanied by reduction of the transition metal cations $[19,32]$. The net electric charge in the pseudocapacitive electrode is the sum of the charge stored from the external circuit and that transferred by ion intercalation. On the other hand, the carbon electrode, of thickness $L_{C}$, was located at $L \leq x \leq L+L_{C}$ 
and did not react chemically with the electrolyte.

To make the problem mathematically tractable, the following assumptions were made: (1) the heterogeneous redox reaction expressed by Equation (1) occurred within the Stern layer near the pseudocapacitive electrode. (2) Bulk motion of the electrolyte was negligible. (3) Transport of the intercalated cations in the pseudocapacitive electrode was treated as a diffusion process. (4) No phase transitions occurred in the pseudocapacitive material. (5) The material properties of the electrodes and electrolyte were constant. (6) The Stern layer thickness $H$ was equal to the largest effective ion radius so that $H=\max \left(a_{i} / 2\right)$. (7) Nonelectrostatic ion adsorption was negligible. (8) The Dufour energy flux, i.e., the energy flux due to gradients of electrochemical potential and/or pressure, was negligible [19, 33, 34]. Finally, (9) the temperature was assumed to be uniform within the narrow Stern layers at all times, and (10) the cell was thermally insulated. For simplicity and in the interest of physical interpretation, the governing equations are presented in 1D Cartesian coordinates. The general 3D formulation can be found in the Supplementary Material.

\subsection{Electrochemical transport model}

The governing equations for (i) the local electric potential $\psi(x, t)$ in both electrodes and in the electrolyte, (ii) the local concentrations $c_{i, E}(x, t)$ of ion species $i$ in the diffuse layer of the electrolyte, and (iii) the local intercalated $\mathrm{Li}^{+}$concentration $c_{1, P}(x, t)$ in the pseudocapacitive electrode for a hybrid pseudocapacitor were previously presented in Ref. [25] and need not

be discussed in detail. Instead, the equations are summarized in Table 1. The subscripts $P$, $E$, and $C$ denote quantities within the pseudocapacitive electrode, the electrolyte, and the carbon electrode, respectively. In the electrodes, the electric potential $\psi(x, t)$ obeys Ohm's law while the $\mathrm{Li}^{+}$concentration $c_{1, P}(x, t)$ obeys the mass conservation equation and Fick's law of diffusion. Here, $\sigma_{P}$ and $\sigma_{C}$ are the electrical conductivities of the pseudocapacitive and carbon electrodes, respectively, while the diffusion coefficient $\left(\right.$ in $\left.\mathrm{m}^{2} \mathrm{~s}^{-1}\right)$ for the intercalated $\mathrm{Li}^{+}$in the pseudocapacitive electrode material is denoted by $D_{1, P}$. In the electrolyte, $\psi(x, t)$ and $c_{i, E}(x, t)$ are governed by the generalized modified Poisson-Nernst-Planck (GMPNP) model [35] accounting for finite ion size and for multi-species and/or asymmetric electrolytes, 
Table 1: One-dimensional governing equations for electric potential $\psi(x, t)$, ion concentrations $c_{i, E}(x, t)$, and reduced $\mathrm{Li}^{+}$concentration $c_{1, P}(x, t)$ in the pseudocapacitive and carbon electrodes and in the electrolyte $[25]$.

\begin{tabular}{|llll|}
\hline Variable & $\begin{array}{l}\text { Pseudocapacitive } \\
\text { electrode }\end{array}$ & Electrolyte & $\begin{array}{l}\text { Carbon } \\
\text { electrode }\end{array}$ \\
\hline $\begin{array}{l}\text { Electric potential } \\
\psi(x, t)\end{array}$ & $\frac{\partial}{\partial x}\left(\sigma_{P} \frac{\partial \psi}{\partial x}\right)=0$ & $-\frac{\partial}{\partial x}\left(\epsilon_{0} \epsilon_{r} \frac{\partial \psi}{\partial x}\right)=F \sum_{i=1}^{n} z_{i, E} c_{i, E}$ & $\frac{\partial}{\partial x}\left(\sigma_{C} \frac{\partial \psi}{\partial x}\right)=0$ \\
\hline $\begin{array}{l}\text { Ion concentrations } \\
c_{i, E}(x, t)\end{array}$ & $\mathrm{N} / \mathrm{A}$ & $\frac{\partial c_{i, E}}{\partial t}=-\frac{\partial N_{i, E}}{\partial x}$ & $\mathrm{~N} / \mathrm{A}$ \\
\hline $\begin{array}{l}\text { Intercalated Li } \\
\text { concentration } \\
c_{1, P}(x, t)\end{array}$ & $\frac{\partial c_{1, P}}{\partial t}=\frac{\partial}{\partial x}\left(D_{1, P} \frac{\partial c_{1, P}}{\partial x}\right)$ & $\mathrm{N} / \mathrm{A}$ & $\mathrm{N} / \mathrm{A}$ \\
\hline
\end{tabular}

i.e., those with more than two ion species $i$ and/or having different valencies $z_{i, E}$, effective ion diameters $a_{i, E}$, and/or diffusion coefficients $D_{i, E}[25,35]$. Here, $\epsilon_{0}=8.854 \times 10^{-12} \mathrm{~F} \mathrm{~m}^{-1}$ and $\epsilon_{r}$ are respectively the vacuum permittivity and the relative permittivity of the electrolyte. The Faraday constant is denoted by $F=96485 \mathrm{C} \mathrm{mol}^{-1}$ and $n$ is the number of ion species in the electrolyte. The flux $N_{i, E}$ of ions in the diffuse layer of the electrolyte is expressed, in $1 \mathrm{D}$, as $[25,35]$

$$
N_{i, E}=-D_{i, E} \frac{\partial c_{i, E}}{\partial x}-\frac{D_{i, E} z_{i, E} F c_{i, E}}{R_{u} T} \frac{\partial \psi}{\partial x}-\frac{D_{i, E} c_{i, E} N_{A}}{1-N_{A} \sum_{j=1}^{n} a_{j, E}^{3} c_{j, E}} \sum_{j=1}^{n} a_{j, E}^{3} \frac{\partial c_{j, E}}{\partial x} .
$$

Here, $T$ is the local absolute temperature (in $\mathrm{K}$ ), $N_{A}=6.022 \times 10^{23} \mathrm{~mol}^{-1}$ is the Avogadro constant, and $R_{u}=8.314 \mathrm{~J} \mathrm{~K}^{-1} \mathrm{~mol}^{-1}$ is the universal gas constant. The first and second terms on the right-hand side of Equation (2) represent ion fluxes due to diffusion and electromigration, respectively. The third term accounts for steric effects so that the ion concentration cannot exceed $c_{i, E, \max }=1 / N_{A} a_{i, E}^{3}$ corresponding to simple cubic packing of spherical ions [35].

\subsubsection{Faradaic and capacitive current densities}

At the interface between the pseudocapacitive electrode and the electrolyte $(x=-L)$, the total current density $j_{T}(t)$ can be divided into a faradaic contribution $j_{F}(t)$ resulting from ion intercalation and a capacitive contribution $j_{C}(t)$ resulting from EDL formation, so 
that $j_{T}(t)=j_{F}(t)+j_{C}(t)$. Here, $j_{F}(t)$ is described by the generalized Frumkin-Butler-Volmer model expressed as $[17,19,25,36,37]$

$$
j_{F}(t)=j_{F, 0}(t)\left\{\exp \left[\frac{(1-\alpha) z_{1, E} F \eta(t)}{R_{u} T}\right]-\exp \left[\frac{-\alpha z_{1, E} F \eta(t)}{R_{u} T}\right]\right\} .
$$

The surface overpotential $\eta(t)=\Delta \psi_{H}(t)-\Delta \psi_{e q}(t)$ (in V) represents the deviation of the electric potential drop across the Stern layer $\Delta \psi_{H}(t)=\psi(-L, t)-\psi(-L+H, t)$ from its value at equilibrium $\Delta \psi_{e q}[19,25]$. The transfer coefficient is denoted by $\alpha$. The exchange current density $j_{F, 0}(t)$ is given by $[25,38,39]$

$$
j_{F, 0}(t)=z_{1, E} F k_{0} c_{1, E}(-L+H, t)^{1-\alpha}\left[c_{1, P, \max }-c_{1, P}(-L, t)\right]^{\alpha} c_{1, P}(-L, t)^{\alpha}
$$

where $k_{0}$ is the reaction rate constant in $\mathrm{m}^{1+3 \alpha} \mathrm{mol}^{-\alpha} \mathrm{s}^{-1}$ and $c_{1, P, \text { max }}$ is the theoretical maximum concentration of intercalated $\mathrm{Li}^{+}$in the pseudocapacitive electrode. Here, $\mathrm{Li}^{+}$intercalation corresponds to negative faradaic current density, i.e., $j_{F}<0 \mathrm{~mA} \mathrm{~cm}^{-2}$, and the transfer coefficient is taken as $\alpha=1 / 2$, corresponding to identical energy barriers for the forward and backward redox reactions $[17,19]$. The capacitive current density $j_{C}(t)$ corresponds to the displacement current in the Stern layer and is defined as

$$
j_{C}(t)=-\epsilon_{0} \epsilon_{r} \frac{\partial^{2} \psi}{\partial x \partial t}(-L+H, t)
$$

\subsubsection{Analytical expressions in asymptotic regimes}

In the asymptotic faradaic and capacitive regimes, simpler analytical expressions have been derived for the intercalated $\mathrm{Li}^{+}$concentration $c_{1, P}(x, t)$ and the surface overpotential $\eta(t)[25]$. They were derived for a 1D hybrid pseudocapacitor cycled galvanostatically with imposed current density $j_{i m}(t)= \pm j_{s}$ at oscillatory steady state and assuming that the pseudocapacitive electrode was thin enough that the intercalated $\mathrm{Li}^{+}$concentration therein remained uniform. These expressions can also be useful for interpreting the thermal behavior. In the faradaic regime, the uniform intercalated $\mathrm{Li}^{+}$concentration $c_{1, P, F}(t)$ in the 
pseudocapacitive electrode varies linearly according to [25]

$$
c_{1, P, F}(t)=c_{1, P, 0}+(-1)^{p} \begin{cases}\frac{j_{s} t_{c}}{z_{1, E} F L_{P}}\left(\frac{t}{t_{c}}-n_{c}+1\right) & \text { for charging } \\ \frac{j_{s} t_{c}}{z_{1, E} F L_{P}}\left(n_{c}-\frac{t}{t_{c}}\right) & \text { for discharging }\end{cases}
$$

where $p=0$ for cells charged by $\mathrm{Li}^{+}$intercalation and $p=1$ for those charged by $\mathrm{Li}^{+}$ deintercalation while $n_{c}$ is the cycle number and $t_{c}$ the cycle period required for the cell potential $\psi_{s}$ to cycle from $\psi_{\min }$ to $\psi_{\max }$ and back. The corresponding overpotential $\eta_{F}(t)$ in this regime is expressed as [25]

$$
\eta_{F}(t)=\frac{2 R_{u} T}{z_{1, E} F} \sinh ^{-1}\left[\frac{j_{i m}(t)}{2 z_{1, E} F k_{0} \sqrt{c_{1, E, \infty}} \sqrt{c_{1, P, \max }-c_{1, P, F}(-L, t)} \sqrt{c_{1, P, F}(-L, t)}}\right] .
$$

In the capacitive regime, the $\mathrm{Li}^{+}$concentration at oscillatory steady state remains approximately constant such that $c_{1, P}(x, t) \approx c_{1, P, C}$ and the overpotential $\eta_{C}(t)$ varies linearly with slope $[25]$

$$
\frac{\mathrm{d} \eta_{C}(t)}{\mathrm{d} t}=\frac{j_{s} H}{\epsilon_{0} \epsilon_{r}}
$$

\subsection{Thermal model}

The governing equation for the temperature and the local heat generation rates in each region of the hybrid pseudocapacitor can be derived from energy conservation principles applied to a fixed control volume $[11,12]$. The temperature is governed by the heat diffusion equation expressed as $[11,12,40]$

$$
\rho c_{p} \frac{\partial T}{\partial t}=\frac{\partial}{\partial x}\left(k \frac{\partial T}{\partial x}\right)+\dot{q}
$$

Here, $\rho, c_{p}$, and $k$ are the density, specific heat, and thermal conductivity of the material, respectively. They will be denoted with subscripts $P, E$, and $C$ in the pseudocapacitive electrode, in the electrolyte, and in the carbon electrode, respectively.

\subsubsection{Heat generation rates in the electrodes and electrolyte diffuse layer}

In the electrodes and in the diffuse layer, there is negligible bulk motion and Dufour energy flux [Assumptions (2) and (8)]. The general mass conservation equation accounting 
for homogeneous chemical reactions can be expressed as [19]

$$
\frac{\partial c_{i}}{\partial t}=-\frac{\partial N_{i}}{\partial x}+\dot{r}_{i}
$$

where $\dot{r}_{i}$ is the local production rate of species $i\left(\right.$ in $\mathrm{mol} \mathrm{m}^{-3} \mathrm{~s}^{-1}$ ) due to chemical reactions. Then, following the derivation procedure used in Ref. [11] for EDLCs, the local volumetric heat generation rate $\dot{q}(x, t)$ (in $\mathrm{W} \mathrm{m}^{-3}$ ) can be expressed as

$$
\dot{q}=-\sum_{i=0}^{n+1} N_{i} \frac{\partial \bar{H}_{i}}{\partial x}-\sum_{i=0}^{n+1} \bar{H}_{i} \dot{r}_{i}
$$

where $\bar{H}_{i}$ is the partial molar enthalpy of ion species $i(1 \leq i \leq n)$ or of the electrons, corresponding to $i=n+1$. The first term on the right-hand side of Equation (11) is the same as that derived for EDLCs $[11,12]$. It is the sum of the heat generation rates (i) $\dot{q}_{E}$ arising from electric charge carriers (ions or electrons) decreasing their electrical potential energy and (ii) the "heat of mixing" $\dot{q}_{S}$ arising from fluxes of ions, $\mathrm{Li}^{+}$, and/or electrons along gradients of chemical potential, partial molar entropy, and temperature [11, 41]. The electrical heat generation rate $\dot{q}_{E}$ is defined as $[11,12]$

$$
\dot{q}_{E}=E j
$$

where $E=-\partial \psi / \partial x$ is the electric field and $j$ is the current density arising from the flow of electrons or ions [19]. The heat of mixing heat generation rate $\dot{q}_{S}$ is defined as $[11,12]$

$$
\dot{q}_{S}=\sum_{i=1}^{n+1} N_{i, j} R_{u} \frac{\partial}{\partial x}\left(T^{2} \frac{\partial \ln \gamma_{i, j}}{\partial T}\right) \quad \text { in region } j=P, E, \text { or } C
$$

where $\gamma_{i}$ is the activity coefficient of ion species $i(=1,2 \ldots n)$ or of electrons $i=n+1$. Finally, the second term on the right-hand side of Equation (11) corresponds to heat generated by homogeneous chemical reactions.

In the $1 D$ electrodes. The current density $j$ results from the flux of electrons and is related to the electric field $E$ by Ohm's law expressed as $j=\sigma_{P} E$ in the pseudocapacitive electrode and $j=\sigma_{C} E$ in the carbon electrode [19]. Here, the transport of intercalated $\mathrm{Li}^{+}$ions 
is accompanied by equal numbers of electrons according to Equation (1) and thus does not affect the net electric current. Thus, the electrical heat generation rate $\dot{q}_{E}$ reduces to irreversible Joule heating expressed as

$$
\dot{q}_{E}=\dot{q}_{J, i r r}= \begin{cases}\frac{j^{2}}{\sigma_{P}} & \text { in the pseudocapacitive electrode } \\ \frac{j^{2}}{\sigma_{C}} & \text { in the carbon electrode. }\end{cases}
$$

The heat of mixing due to the 1D electron flux $N_{n+1, P}=-j / F$ and to the 1D flux of intercalated $\mathrm{Li}^{+} N_{1, P}=-D_{1, P} \partial c_{1, P} / \partial x$ could potentially produce a reversible heat generation rate in the electrode expressed as

$$
\dot{q}_{S}=-R_{u}\left\{\frac{j}{F} \frac{\partial}{\partial x}\left[T^{2} \frac{\partial \ln \left(\gamma_{n+1, P}\right)}{\partial T}\right]+D_{1, P} \frac{\partial c_{1, P}}{\partial x} \frac{\partial}{\partial x}\left[T^{2} \frac{\partial \ln \left(\gamma_{1, P}\right)}{\partial T}\right]\right\}
$$

The present study neglects $\dot{q}_{S}$ in the electrodes. Indeed, the first term associated with the electrons is expected to be negligible as (i) the EDL region in an electrode is negligibly thin [18] and (ii) reversible heating in the electrode has not been reported for other applications such as dielectric capacitors where this expression would also apply. The second term on the right-hand side of Equation (15) was neglected as the required expression for the activity coefficient $\gamma_{1, P}$ of intercalated $\mathrm{Li}^{+}$as a function of temperature and of $\mathrm{Li}^{+}$concentration inside the pseudocapacitive material is, to the best of our knowledge, not available in the literature.

Finally, in the pseudocapacitive electrode, the heat generation rate $\dot{q}_{F, P}(x, t)=$ $\sum_{i=0}^{n+1}\left(\bar{H}_{i, P} \dot{r}_{i, P}\right)(x, t)$ arises as intercalated $\mathrm{Li}^{+}$ions move from one lattice site to the next. Here, the local rate of $\mathrm{Li}_{m} \mathrm{M}_{p} \mathrm{O}_{p}$ production can be related to the concentration $c_{1, P}$ predicted by the electrochemical transport model as $(1 / m) \partial c_{1, P} / \partial t$. In addition, the production rates $\dot{r}_{i, P}$ are related by stoichiometry. As a result, the heat generation rate $\dot{q}_{F, P}$ can be expressed as

$$
\dot{q}_{F, P}(x, t)=\dot{q}_{F, P, r e v}(x, t)=-\frac{\Delta H_{r}}{m} \frac{\partial c_{1, P}}{\partial t}
$$

where $\Delta H_{r}=\bar{H}_{\mathrm{Li}_{m} \mathrm{M}_{p} \mathrm{O}_{q}}-\bar{H}_{\mathrm{M}_{p} \mathrm{O}_{q}}-m \bar{H}_{\mathrm{Li}^{+}}-m \bar{H}_{\mathrm{e}^{-}}$is the enthalpy of reaction corresponding to Equation (1), i.e., the enthalpy change resulting from the production of one mole of $\mathrm{Li}_{m} \mathrm{M}_{p} \mathrm{O}_{q}$ 
$[42,43]$. Since $\partial c_{1, P} / \partial t$ changes sign based on the direction of the reaction while $\Delta H_{r}$ does not, $\dot{q}_{F, P}$ is a reversible heat generation rate. Note that, for constant $\Delta H_{r}$, the total contribution of $\dot{q}_{F, P}(x, t)$ in the electrode $\int_{-L_{P}-L}^{-L} \dot{q}_{F, P}(x, t) d x=-\left(\Delta H_{r} / m\right) \frac{\partial}{\partial t} \int_{-L_{P}-L}^{-L} c_{1, P}(x, t) d x$ is proportional to the faradaic current density $j_{F}(t)$.

In the diffuse layer. The physical processes causing heat generation in the diffuse layer of hybrid pseudocapacitors are identical to those taking place in EDLCs and $\dot{q}_{F}$ is absent, since no chemical reactions occur there [Assumption (1)]. Thus, our previous model can be used [12]. In brief, for an electrolyte with ionic current density $j=\sum_{i=1}^{n} z_{i, E} F N_{i, E}[19]$ and obeying the above GMPNP model, $\dot{q}_{E}$ in the diffuse layer consists of three contributions such that $\dot{q}_{E}=\dot{q}_{J, i r r}+\dot{q}_{E, d}+\dot{q}_{E, s}[12]$. The irreversible Joule heat generation rate $\dot{q}_{J, i r r}$ is expressed in $1 \mathrm{D}$ as $[12]$

$$
\dot{q}_{J, i r r}=\frac{j^{2}}{\sigma_{E}}
$$

where $\sigma_{E}$ is the local electrical conductivity of the electrolyte (in $\mathrm{S} \mathrm{m}^{-1}$ ) and can be expressed for the GMPNP model as $\sigma_{E}=\left(F^{2} / R_{u} T\right) \sum_{i=1}^{n} z_{i, E}^{2} D_{i, E} c_{i, E}$ [19]. Then, the reversible diffusion and steric contributions $\dot{q}_{E, d}$ and $\dot{q}_{E, s}$ are expressed as [12]

$$
\begin{aligned}
\dot{q}_{E, d} & =\frac{j}{\sigma_{E}} F \sum_{i=1}^{n} D_{i, E} z_{i, E} \frac{\partial c_{i, E}}{\partial x} \\
\text { and } \quad \dot{q}_{E, s} & =\frac{j}{\sigma_{E}} N_{A}\left(\sum_{i=1}^{n} a_{i, E}^{3} \frac{\partial c_{i, E}}{\partial x}\right) \frac{F \sum_{i=1}^{n} D_{i, E} z_{i, E} c_{i, E}}{1-N_{A} \sum_{i=1}^{n} a_{i, E}^{3} c_{i, E}} .
\end{aligned}
$$

They are non-zero only in the presence of an ion concentration gradient, i.e., near the electrode/electrolyte interfaces.

Evaluating the heat of mixing $\dot{q}_{S}$ in the diffuse layer requires an expression for the ions' activity coefficients $\gamma_{i, E}$. The present study used the Debye-Hückel limiting law due to its theoretical derivation depending on temperature and ion concentrations [11]. Then, the ion 
activity coefficient $\gamma_{i, E}$ in the diffuse layer is expressed as $[19,44]$

$$
\ln \gamma_{i, E}=-\frac{z_{i, E}^{2} e F^{2}\left(\sum_{j=1}^{n} z_{j, E}^{2} c_{j, E}\right)^{1 / 2}}{8 \pi\left(\epsilon_{0} \epsilon_{r} R_{u} T\right)^{3 / 2}} .
$$

Assuming negligible contribution from the solvent, corresponding to species $i=0$, the heat of mixing $\dot{q}_{S}$ comprises two terms expressed as [12]

$$
\begin{aligned}
\dot{q}_{S, c} & =\frac{3}{32 \pi} \frac{e F^{2}}{\left(\epsilon_{0} \epsilon_{r}\right)^{3 / 2}\left(R_{u} T \sum_{i=1}^{n} z_{i, E}^{2} c_{i, E}\right)^{1 / 2}}\left(\sum_{i=1}^{n} z_{i, E}^{2} N_{i, E}\right)\left(\sum_{i=1}^{n} z_{i, E}^{2} \frac{\partial c_{i, E}}{\partial x}\right) \\
\text { and } \quad \dot{q}_{S, T} & =-\frac{3}{32 \pi} \frac{e F^{2}\left(\sum_{i=1}^{n} z_{i, E}^{2} c_{i, E}\right)^{1 / 2}}{\left(\epsilon_{0} \epsilon_{r}\right)^{3 / 2} R_{u}^{1 / 2} T^{3 / 2}}\left(\sum_{i=1}^{n} z_{i, E}^{2} N_{i, E}\right) \frac{\partial T}{\partial x} .
\end{aligned}
$$

They are such that $\dot{q}_{S}=\dot{q}_{S, c}+\dot{q}_{S, T}$ in the diffuse layer [12].

\subsubsection{Stern layers}

The temperature across the Stern layer was assumed to be uniform due to its small thickness. Then, in the absence of ion or solvent accumulation, the energy conservation equation for either Stern layer can be expressed as

$$
\frac{\partial\left(\rho_{E} u\right)}{\partial t}=\rho_{E} c_{p, E} \frac{\partial T}{\partial t}=\frac{q_{i n}^{\prime \prime}-q_{o u t}^{\prime \prime}}{H}
$$

Here, $u$ is the specific internal energy of the electrolyte in the Stern layer while $q_{i n}^{\prime \prime}$ and $q_{\text {out }}^{\prime \prime}$ are the energy fluxes into and out of the Stern layer, respectively. Near the carbon electrode, the energy fluxes $q_{i n}^{\prime \prime}$ and $q_{\text {out }}^{\prime \prime}$ are solely due to heat conduction. Then, the energy conservation equation can be written as

$$
\rho_{E} c_{p, E} \frac{\partial T}{\partial t}=\frac{1}{H}\left[k_{C} \frac{\partial T}{\partial x}(L, t)-k_{E} \frac{\partial T}{\partial x}(L-H, T)\right]
$$

Near the pseudocapacitive electrode, the energy fluxes result not only from heat conduction but also from chemical reactants entering and exiting the Stern layer. Assuming negligible Dufour effect, the local energy fluxes $q_{i n}^{\prime \prime}(t)=q^{\prime \prime}(-L, t)$ at the electrode/electrolyte interface 
and $q_{\text {out }}^{\prime \prime}(t)=q^{\prime \prime}(-L+H, t)$ at the Stern/diffuse layer interface can be expressed as [19]

$$
\begin{aligned}
q_{i n}^{\prime \prime}(t) & =-k_{P} \frac{\partial T}{\partial x}(-L, t)+\sum_{i=1}^{n+1}\left(\bar{H}_{i, P} N_{i, P}\right)(-L, t) \\
\text { and } \quad q_{\text {out }}^{\prime \prime}(t) & =-k_{E} \frac{\partial T}{\partial x}(-L+H, t)+\sum_{i=1}^{n}\left(\bar{H}_{i, E} N_{i, E}\right)(-L+H, t) .
\end{aligned}
$$

Then, in the Stern layer near the pseudocapacitive electrode, Equation (23) becomes

$$
\rho_{E} c_{p, E} \frac{\partial T}{\partial t}=\frac{1}{H}\left[k_{E} \frac{\partial T}{\partial x}(-L+H, t)-k_{P} \frac{\partial T}{\partial x}(-L, t)\right]+\dot{q}_{F, E}(t)
$$

where $\dot{q}_{F, E}$ is the heat generation rate due to the faradaic reaction given by

$$
\dot{q}_{F, E}(t)=-\frac{1}{H}\left[\sum_{i=1}^{n}\left(\bar{H}_{i, E} N_{i, E}\right)(-L+H, t)-\sum_{i=1}^{n+1}\left(\bar{H}_{i, P} N_{i, P}\right)(-L, t)\right] .
$$

The only non-zero terms in Equation (28) correspond to species involved in the redox reaction [Equation (1)] because no other species enter or exit the Stern layer. The fluxes $N_{i, E}$ and $N_{i, P}$ of all reacting species are related to the electron flux $N_{n+1, P}$ and the faradaic current density $j_{F}$ by stoichiometry [19]. Let us consider the general redox reaction occurring at the pseudocapacitive electrode/electrolyte interface $\sum_{i=0}^{n+1}\left(s_{i, P} M_{i, P}+s_{i, E} M_{i, E}\right) \rightleftharpoons 0$, where $s_{i}$ is the stoichiometric coefficient for reactant $M_{i}$ and subscripts $P$ and $E$ respectively refer to the pseudocapacitive electrode and electrolyte. For this reaction, the fluxes of reactants $N_{i, E}$ in the electrolyte and $N_{i, P}$ in the pseudocapacitive electrode are related by $j_{F}(t) / F=$ $-N_{n+1, P}(-L, t)=-\left(s_{n+1, P} / s_{i, P}\right) N_{i, P}(-L, t)=\left(s_{n+1, P} / s_{i, E}\right) N_{i, E}(-L+H, t)$. Then, $\dot{q}_{F, E}$ can be expressed as

$$
\dot{q}_{F, E}=-\frac{j_{F}(t)}{H F s_{n+1, P}} \sum_{i=1}^{n+1}\left[s_{i, E} \bar{H}_{i, E}(-L+H, t)+s_{i, P} \bar{H}_{i, P}(-L, t)\right] .
$$

Expressing the partial molar enthalpy as $\bar{H}_{i}=\tilde{\mu}_{i}+T \bar{S}_{i}[45]$ yields

$$
\begin{aligned}
\dot{q}_{F, E}=-\frac{j_{F}(t)}{H} & \left\{\frac{1}{s_{n+1, P} F} \sum_{i=1}^{n+1}\left[s_{i, E} \tilde{\mu}_{i, E}(-L+H, t)+s_{i, P} \tilde{\mu}_{i, P}(-L, t)\right]\right. \\
& \left.+\frac{1}{s_{n+1, P} F} T \sum_{i=1}^{n+1}\left[s_{i, E} \bar{S}_{i, E}(-L+H, t)+s_{i, P} \bar{S}_{i, P}(-L, t)\right]\right\} .
\end{aligned}
$$


The two terms within the brackets correspond to expressions for the surface overpotential $\eta$ and the Peltier coefficient $\Pi$, respectively defined as [19]

$$
\eta=-\sum_{i=0}^{n+1} \frac{\left(s_{i, P} \tilde{\mu}_{i, P}+s_{i, E} \tilde{\mu}_{i, E}\right)}{s_{n+1, P} F} \quad \text { and } \quad \Pi=-\frac{T}{F} \sum_{i=0}^{n+1} \frac{\left(s_{i, P} \bar{S}_{i, P}+s_{i, E} \bar{S}_{i, E}\right)}{s_{n+1, P}}
$$

Then, $\dot{q}_{F, E}(t)$ can be divided into one irreversible $\dot{q}_{F, E, \text { irr }}$ and one reversible $\dot{q}_{F, E, \text { rev }}$ contribution such that $\dot{q}_{F, E}=\dot{q}_{F, E, i r r}+\dot{q}_{F, E, \text { rev }}$ with the remarkably simple expressions

$$
\dot{q}_{F, E, i r r}(t)=\frac{j_{F}(t)}{H} \eta(t) \quad \text { and } \quad \dot{q}_{F, E, r e v}(t)=\frac{j_{F}(t)}{H} \Pi(t) .
$$

These heat generation rates were confined to the Stern layer adjacent to the pseudocapacitive electrode, i.e., $-L \leq x \leq-L+H$. Note that the form of the heat generation rates defined by Equation (32) was also derived in Ref. [19] for an electrochemical reaction at steady state but ignoring EDL formation.

Here, $\dot{q}_{F, E, \text { rev }}$ generated at the electrode/electrolyte interface [Equation (32)] and the total heat generation rate due to $\dot{q}_{F, P, \text { rev }}$ inside the pseudocapacitive electrode [Equation (16)] are both proportional to $j_{F}$. Experimentally, it would be difficult to distinguish between them to measure separate values for $\Pi$ and $\Delta H_{r}$. Indeed, to the best of our knowledge, the data required to evaluate $\Delta H_{r}$ for the homogeneous reaction inside the electrode is not available in the literature. As a result, the present study simulates $\dot{q}_{F, E \text {,rev }}$ inside the Stern layer only and neglects $\dot{q}_{F, P, r e v}$.

To summarize, Table 2 gives the expressions for the irreversible and reversible heat generation rates to be combined with Equation (9) in each region of the 1D hybrid pseudocapacitor simulated in the present study.

\subsection{Initial and boundary conditions}

Each governing equation for $\psi(x, t), c_{1, E}(x, t), c_{2, E}(x, t), c_{1, P}(x, t)$, and $T(x, t)$ requires one initial condition and two boundary conditions in each region where it is solved. Here, the $\mathrm{Li}^{+}$concentration $c_{1, P}(x, t)$ was computed only in the pseudocapacitive electrode, and the ion concentrations $c_{1, E}(x, t)$ and $c_{2, E}(x, t)$ only in the diffuse layer. Meanwhile, the potential $\psi(x, t)$ and the temperature $T(x, t)$ were computed in the pseudocapacitive and 
Table 2: Summary of the irreversible and reversible heat generation rates $\dot{q}_{i r r}$ and $\dot{q}_{\text {rev }}$ simulated in each region for the $1 \mathrm{D}$ hybrid pseudocapacitor illustrated in Figure 1 such that $\dot{q}=\dot{q}_{i r r}+\dot{q}_{r e v}$.

\begin{tabular}{|lccc|}
\multicolumn{1}{c}{ Region } & Coordinates & $\begin{array}{c}\text { Irreversible heat } \\
\text { generation rate } \\
\left(\mathbf{W} \mathbf{~ m}^{-3}\right)\end{array}$ & $\begin{array}{c}\text { Reversible heat } \\
\text { generation rate } \\
\left(\mathbf{W} \mathbf{~ m}^{-3}\right)\end{array}$ \\
\hline $\begin{array}{l}\text { Pseudocapacitive } \\
\text { electrode }\end{array}$ & $-L_{P}-L \leq x<-L$ & $\dot{q}_{i r r}=\frac{j^{2}}{\sigma_{P}}$ & $\mathrm{~N} / \mathrm{A}$ \\
\hline $\begin{array}{l}\text { Pseudocapacitive } \\
\text { electrode Stern } \\
\text { layer }\end{array}$ & $-L \leq x<-L+H$ & $\dot{q}_{i r r}=\frac{j_{F} \eta}{H}$ & $\dot{q}_{r e v}=\frac{j_{F} \Pi}{H}$ \\
\hline $\begin{array}{l}\text { Electrolyte } \\
\text { diffuse layer }\end{array}$ & $-L+H \leq x<L-H$ & $\dot{q}_{i r r}=\frac{j^{2}}{\sigma_{E}}$ & $\dot{q}_{r e v}=\dot{q}_{E, d}+\dot{q}_{E, s}+\dot{q}_{S, c}+\dot{q}_{S, T}$ \\
\hline $\begin{array}{l}\text { Carbon electrode } \\
\text { Stern layer }\end{array}$ & $L-H \leq x<L$ & $\mathrm{~N} / \mathrm{A}$ & $\mathrm{N} / \mathrm{A}$ \\
\hline Carbon electrode & $L \leq x<L+L_{C}$ & $\dot{q}_{i r r}=\frac{j^{2}}{\sigma_{C}}$ & $\mathrm{~N} / \mathrm{A}$ \\
\hline
\end{tabular}

carbon electrodes as well as the diffuse layer of the electrolyte. The potential $\psi$ and temperature $T$ inside the Stern layers were not explicitly simulated. Instead, the Stern layers and the faradaic heat generation rate $\dot{q}_{F, E}$ therein were accounted for by using the boundary conditions at the electrode/electrolyte and at the Stern/diffuse layer interfaces.

\subsubsection{Initial conditions}

Initially, the potential was taken as uniform and equal to zero across the entire device such that

$$
\psi(x, 0)=0 \mathrm{~V} .
$$

The initial concentration of $\mathrm{Li}^{+}$in the pseudocapacitive electrode was uniform and equal to $c_{1, P, 0}$, i.e.,

$$
c_{1, P}(x, 0)=c_{1, P, 0}
$$


Table 3: Boundary conditions for electric potential $\psi(x, t)$ and concentrations $c_{1, E}(x, t)$ and $c_{2, E}(x, t)$ of ions in the electrolyte and $c_{1, P}(x, t)$ of $\mathrm{Li}^{+}$in the pseudocapacitive electrode. [25].

\begin{tabular}{|lcc|}
\multicolumn{1}{c}{ Region } & $\begin{array}{c}\text { Electric potential } \\
\boldsymbol{\psi}(\boldsymbol{x}, \boldsymbol{t})\end{array}$ & $\begin{array}{c}\text { Ion concentrations } \\
\boldsymbol{c}_{\boldsymbol{I}}(\boldsymbol{x}, \boldsymbol{t}) \text { and } \boldsymbol{c}_{2}(\boldsymbol{x}, \boldsymbol{t})\end{array}$ \\
\hline $\begin{array}{l}\text { Pseudocapacitive } \\
\text { electrode } \\
-\boldsymbol{L}-\boldsymbol{L}_{P} \leq \boldsymbol{x} \leq-\boldsymbol{L}\end{array}$ & $-\sigma_{P} \frac{\partial \psi}{\partial x}\left(-L-L_{P}, t\right)=j_{i m}(t)$ & $N_{1, P}\left(-L-L_{P}, t\right)=0$ \\
\hline $\begin{array}{l}\text { Electrolyte diffuse } \\
\text { layer } \\
-\boldsymbol{L}+\boldsymbol{H} \leq \boldsymbol{x} \leq \boldsymbol{L}-\boldsymbol{H}\end{array}$ & $\frac{\partial \psi}{\partial x}(-L+H, t)=\frac{\psi(-L+H, t)-\psi(-L, t)}{H}$ & $N_{1, P}(-L, t)=\frac{j_{F}(t)}{F}$ \\
\hline $\begin{array}{l}\text { Carbon electrode } \\
\boldsymbol{L} \leq \boldsymbol{x} \leq \boldsymbol{L}+\boldsymbol{L}_{C}\end{array}$ & $-\sigma_{C} \frac{\partial \psi}{\partial x}(L, t)=-\epsilon_{0} \epsilon_{r} \frac{\partial \psi}{\partial x}(L-H, t)$ & $N_{2, E}(-L+H, t)=\frac{j_{F}(t)}{F}$ \\
\hline & $\psi$ & $N_{1, E}(L-H, t)=0$ \\
\hline
\end{tabular}

Similarly, the initial ion concentrations in the diffuse layer of the electrolyte were uniform with bulk concentrations $c_{i, E, \infty}$ satisfying electroneutrality, i.e.,

$$
c_{i, E}(x, 0)=c_{i, E, \infty} \quad \text { with } \quad \sum_{i=1}^{n} z_{i} c_{i, E, \infty}=0 .
$$

Finally, the initial temperature was uniform and equal to $T_{0}$ throughout the device so that

$$
T(x, 0)=T_{0}
$$

\subsubsection{Boundary conditions}

For the sake of completeness, Table 3 summarizes the boundary conditions for the potential $\psi(x, t)$ and the concentrations $c_{1, P}(x, t), c_{1, E}(x, t)$, and $c_{2, E}(x, t)$ during galvanostatic cycling under current density $\pm j_{s}$. They were identical to those used in Ref. [25]. For galvanostatic cycling over a fixed potential window $\psi_{\min } \leq \psi_{s} \leq \psi_{\max }$ with charging corresponding to $\mathrm{Li}^{+}$deintercalation, $j_{i m}(t)$ was expressed as [25]

$$
j_{i m}(t)= \begin{cases}j_{s} & \text { if } \psi_{\min }<\psi_{s}<\psi_{\max } \text { and } \mathrm{d} \psi_{s} / \mathrm{d} t \geq 0 \text { or } \psi_{s}=\psi_{\min } \\ -j_{s} & \text { if } \psi_{\min }<\psi_{s}<\psi_{\max } \text { and } \mathrm{d} \psi_{s} / \mathrm{d} t<0 \text { or } \psi_{s}=\psi_{\max }\end{cases}
$$


In this method, the duration of the charging and discharging steps was allowed to vary, as implemented experimentally.

Moreover, the pseudocapacitive electrode was thermally insulated at the electrode/current collector interface such that

$$
-k_{P} \frac{\partial T}{\partial x}\left(-L-L_{P}, t\right)=0 \mathrm{~W} \mathrm{~m}^{-2}
$$

The temperature at the electrode/electrolyte interface was equal to that at the Stern/diffuse layer interface [Assumption (9)], i.e.,

$$
T(-L, t)=T(-L+H, t)
$$

The heat flux at the Stern/diffuse layer interface was obtained from the energy conservation Equation (27) so that

$$
-k_{E} \frac{\partial T}{\partial x}(-L+H, t)=-k_{P} \frac{\partial T}{\partial x}(-L, t)+H\left[\dot{q}_{F, E}(t)-\rho_{E} c_{p, E} \frac{\partial T}{\partial t}(-L, t)\right]
$$

Moreover, the temperature was constant across the Stern layer near the carbon electrode so that

$$
T(L-H, t)=T(L, t) .
$$

In the carbon electrode, the heat flux at the electrode/electrolyte interface satisfied Equation (24) such that

$$
-k_{C} \frac{\partial T}{\partial x}(L, t)=-k_{E} \frac{\partial T}{\partial x}(L-H, t)-H \rho_{E} c_{p, E} \frac{\partial T}{\partial t}(L, t)
$$

Finally, the carbon electrode was thermally insulated at the electrode/current collector interface so that

$$
-k_{C} \frac{\partial T}{\partial x}\left(L+L_{C}, t\right)=0 \mathrm{~W} \mathrm{~m}^{-2}
$$

\subsection{Constitutive relationships}

Table 4 summarizes the properties of the electrodes and electrolyte used to solve the electrochemical transport model for $\psi(x, t), c_{1, E}(x, t), c_{2, E}(x, t)$, and $c_{1, P}(x, t)$ for a hybrid 
Table 4: Electrochemical transport properties used for simulating the hybrid pseudocapacitor with binary and asymmetric electrolyte [25].

\begin{tabular}{llllll}
\hline \multicolumn{2}{l}{ Electrodes } & \multicolumn{4}{l}{ Electrolyte } \\
\hline$\sigma_{P}$ & $7 \times 10^{-2}$ & $\mathrm{~S} / \mathrm{m}$ & $\epsilon_{r}$ & 66.1 & \\
$L_{P}$ & 5 & $\mathrm{~nm}$ & $a_{1, E}$ & 0.67 & $\mathrm{~nm}$ \\
$D_{1, P}$ & $10^{-10}$ & $\mathrm{~m}^{2} / \mathrm{s}$ & $a_{2, E}$ & 1.0 & $\mathrm{~nm}$ \\
$c_{1, P, \max }$ & 32.9 & $\mathrm{~mol} / \mathrm{L}$ & $D_{1, E}$ & $2.6 \times 10^{-10}$ & $\mathrm{~m}^{2} / \mathrm{s}$ \\
$\alpha$ & $1 / 2$ & & $D_{2, E}$ & $3.3 \times 10^{-10}$ & $\mathrm{~m}^{2} / \mathrm{s}$ \\
$k_{0}$ & $5 \times 10^{-9}$ & $\mathrm{~m}^{5 / 2} / \mathrm{mol}^{1 / 2} \mathrm{~S}$ & $c_{1, E, \infty}$ & 1 & $\mathrm{~mol} / \mathrm{L}$ \\
$\Delta \psi_{e q}$ & 0 & $\mathrm{~V}$ & $c_{2, E, \infty}$ & 1 & $\mathrm{~mol} / \mathrm{L}$ \\
$\sigma_{C}$ & 100 & $\mathrm{~S} / \mathrm{m}$ & $L$ & 1 & $\mu \mathrm{m}$ \\
$L_{C}$ & 5 & $\mathrm{~nm}$ & & & \\
\hline
\end{tabular}

pseudocapacitor with binary and asymmetric electrolyte. Arbitrary but realistic material properties were chosen for the pseudocapacitive material. Its density $\rho_{P}$ and specific heat $c_{p, P}$ were based on those of $\mathrm{Nb}_{2} \mathrm{O}_{5}$ and taken as $\rho_{P}=4550 \mathrm{~kg} \mathrm{~m}^{-3}[46]$ and $c_{p, P}=496 \mathrm{~J} \mathrm{~kg}^{-1} \mathrm{~K}^{-1}$ [47], respectively. Its thermal conductivity was taken as $k_{P}=10 \mathrm{~W} \mathrm{~m}^{-1} \mathrm{~K}^{-1}$, typical of various metal oxides [48]. The electrolyte simulated was $1 \mathrm{~mol} \mathrm{~L}^{-1} \mathrm{LiClO}_{4}$ in propylene carbonate (PC) solvent, with the $\mathrm{Li}^{+}$and $\mathrm{ClO}_{4}^{-}$ion species denoted by $i=1$ and 2 , respectively. Its density $\rho_{E}=1205 \mathrm{~kg} \mathrm{~m}^{-3}$, specific heat $c_{p, E}=2141 \mathrm{~J} \mathrm{~kg}^{-1} \mathrm{~K}^{-1}$, and thermal conductivity $k_{E}=0.164 \mathrm{~W} \mathrm{~m}^{-1} \mathrm{~K}^{-1}$ were taken as those of PC [49]. The density, specific heat, and thermal conductivity of the carbon electrode were taken as $\rho_{C}=2248 \mathrm{~kg} \mathrm{~m}^{-3}$, $c_{p, C}=710 \mathrm{~J} \mathrm{~kg}^{-1} \mathrm{~K}^{-1}$, and $k_{C}=1.7 \mathrm{~W} \mathrm{~m}^{-1} \mathrm{~K}^{-1}$, respectively [50]. In the absence of reported values for $\mathrm{Li}^{+}$intercalation in pseudocapacitive materials, the Peltier coefficient $\Pi$ was taken as constant and approximated as $\Pi=-0.363 \mathrm{~V}$ based on measurements for the reaction $\mathrm{Li} \rightarrow \mathrm{Li}^{+}+\mathrm{e}^{-}[51]$

Finally, the initial $\mathrm{Li}^{+}$concentration $c_{1, P, 0}$ in the pseudocapacitive electrode was equal to $c_{1, P, 0}=1 \mathrm{~mol} \mathrm{~L}^{-1}$. The initial temperature was $T_{0}=298 \mathrm{~K}$. The cell was cycled galvanostatically at various current densities $j_{s}$ over a fixed potential window with minimum and maximum cell potentials equal to $\psi_{\min }=0 \mathrm{~V}$ and $\psi_{\max }=0.45 \mathrm{~V}$, respectively. 


\subsection{Method of solution}

The one-dimensional governing equations given by Tables 1 and 2 and Equation (9) and the associated initial and boundary conditions were solved numerically using finite element methods. Numerical convergence was assessed based on the predicted potential $\psi(x, t)$, concentrations $c_{1, E}(x, t), c_{2, E}(x, t)$, and $c_{1, P}(x, t)$, and temperature $T(x, t)$. The temperature was the most sensitive to the choice of mesh element size and time step. The mesh element size was the smallest at the Stern/diffuse layer interfaces due to the large potential and concentration gradients in this region and gradually increased away from these boundaries. The mesh was refined by reducing the element size at the Stern/diffuse layer interface and by reducing the maximum element growth rate. The time step was controlled by the relative and absolute time tolerances [52]. At each time step, the estimated local error between the solutions at the previous and the current time step was compared with the time tolerances. The time step was then adjusted until the convergence criterion was satisfied, as described in Ref. [52]. This enabled the use of small time steps during periods of rapid changes in $\psi(x, t), c_{i, E}(x, t), c_{1, P}(x, t)$, and/or $T(x, t)$, while using a larger time step for the rest of the simulation. The numerical solution was considered converged when halving (i) the element size at the Stern/diffuse layer interface, (ii) the maximum element growth rate, and (iii) both the relative and absolute tolerances resulted in less than $0.5 \%$ maximum relative difference in the local temperature rise $T(x, t)-T_{0}$.

Finally, multiple cycles were simulated and an oscillatory steady state in $\psi(x, t), c_{1, E}(x, t)$, $c_{2, E}(x, t)$, and $c_{1, P}(x, t)$ was considered to be reached when the maximum relative difference between each variable's value at time $t$ and its value at time $t-t_{c}$ was less than $1 \%$. For small current densities $j_{s} \lesssim 8 \mathrm{~mA} \mathrm{~cm}^{-2}$, these conditions were typically met by the third cycle. The number of cycles required to reach oscillatory steady state increased with increasing current density $j_{s}$. Note that there was no oscillatory steady state in $T(x, t)$. The temperature continually rose from cycle to cycle because the cell was thermally insulated and subjected to irreversible heating. 


\subsection{Data processing}

It is difficult to directly compare the various local volumetric heat generation rates within the hybrid pseudocapacitor because they act within different sections of the device (Table 2). To assess the contribution of each heat generation rate to the total heat generation, they should be integrated over the entire volume of the cell. Thus, the total Joule heat generation rate $\dot{Q}_{J, i r r}^{\prime \prime}(t)$ per unit electrode surface area (in $\mathrm{W} \mathrm{m}^{-2}$ ) was defined as

$$
\dot{Q}_{J, i r r}^{\prime \prime}(t)=\int_{-L-L_{P}}^{L+L_{C}} \dot{q}_{J, i r r}(x, t) d x=\int_{-L-L_{P}}^{-L} \frac{j^{2}}{\sigma_{P}} d x+\int_{-L+H}^{L-H} \frac{j^{2}}{\sigma_{E}} d x+\int_{L}^{L+L_{C}} \frac{j^{2}}{\sigma_{C}} d x .
$$

It can also be defined based on averaging over each half-cell as

$$
\dot{Q}_{J, i r r}^{\prime \prime}(t)=\int_{-L-L_{P}}^{0} \dot{q}_{J, i r r}(x, t) d x+\int_{0}^{L+L_{C}} \dot{q}_{J, i r r}(x, t) d x=\dot{Q}_{J, i r r, P}^{\prime \prime}(t)+\dot{Q}_{J, i r r, C}^{\prime \prime}(t) .
$$

Similarly, the total irreversible $\dot{Q}_{F, i r r, P}^{\prime \prime}(t)$ and reversible $\dot{Q}_{F, r e v, P}^{\prime \prime}(t)$ faradaic heat generation rates at the pseudocapacitive electrode were defined as

$$
\begin{aligned}
\dot{Q}_{F, i r r, P}^{\prime \prime}(t) & =\int_{-L}^{-L+H} \dot{q}_{F, E, i r r}(t) d x=\dot{q}_{F, E, i r r}(t) H \\
\text { and } \quad \dot{Q}_{F, r e v, P}^{\prime \prime}(t) & =\int_{-L}^{-L+H} \dot{q}_{F, E, r e v}(t) d x=\dot{q}_{F, E, r e v}(t) H .
\end{aligned}
$$

To facilitate comparison with experimental measurements from Ref. [9], the reversible heat generation rates due to EDL formation were computed separately for the pseudocapacitive electrode half-cell $\dot{Q}_{E D L, r e v, P}^{\prime \prime}$ and for the carbon electrode half-cell $\dot{Q}_{E D L, \text { rev }, C}^{\prime \prime}$ and defined as

$$
\begin{aligned}
\dot{Q}_{E D L, r e v, P}^{\prime \prime}(t) & =\int_{-L+H}^{0}\left(\dot{q}_{E, d}+\dot{q}_{E, s}+\dot{q}_{S, c}+\dot{q}_{S, T}\right) d x \\
\text { and } \quad \dot{Q}_{E D L, r e v, C}^{\prime \prime}(t) & =\int_{0}^{L-H}\left(\dot{q}_{E, d}+\dot{q}_{E, s}+\dot{q}_{S, c}+\dot{q}_{S, T}\right) d x .
\end{aligned}
$$


Then, the overall heat generation rate $\dot{Q}_{P}^{\prime \prime}(t)$ from all sources in the pseudocapacitive electrode half-cell was given by

$$
\dot{Q}_{P}^{\prime \prime}(t)=\int_{-L-L_{P}}^{0} \dot{q}(x, t) d x=\dot{Q}_{J, i r r, P}^{\prime \prime}(t)+\dot{Q}_{E D L, r e v, P}^{\prime \prime}(t)+\dot{Q}_{F, \text { irr }, P}^{\prime \prime}(t)+\dot{Q}_{F, r e v, P}^{\prime \prime}(t)
$$

while $\dot{Q}_{C}^{\prime \prime}(t)$ in the carbon electrode half-cell was expressed as

$$
\dot{Q}_{C}^{\prime \prime}(t)=\int_{0}^{L+L_{C}} \dot{q}(x, t) d x=\dot{Q}_{J, i r r, C}^{\prime \prime}(t)+\dot{Q}_{E D L, r e v, C}^{\prime \prime}(t) .
$$

Finally, in order to effectively compare the different heat generation rates, the timeaverage $\overline{\dot{Q}}_{j}^{\prime \prime}$ of heat generation rate $\dot{Q}_{j}^{\prime \prime}(t)$ at oscillatory steady state was defined as

$$
\bar{Q}_{j}^{\prime \prime}=\frac{1}{t_{c}} \int_{\left(n_{c}-1\right) t_{c}}^{n_{c} t_{c}} \dot{Q}_{j}^{\prime \prime}(t) d t
$$

where cycle $n_{c}$ is at oscillatory steady state while subscript $j$ refers to the different heat generation rate contributions.

\section{Results and discussion}

This section presents simulation results for charging the hybrid pseudocapacitor by $\mathrm{Li}^{+}$ deintercalation and cycling over a fixed potential window $0 \mathrm{~V} \leq \psi_{s} \leq 0.45 \mathrm{~V}$ under several current densities $j_{s}$. Predictions for the heat generation rates and temperature are qualitatively compared with experimental measurements reported in the literature $[9,23]$. To better interpret the results, it is informative to consider the electrochemical transport in the device.

\subsection{Electrochemical transport}

Figure 2 shows the cell potential $\psi_{s}(t)=\psi\left(-L-L_{P}, t\right)-\psi\left(L+L_{C}, t\right)$ as a function of time $t-n_{c} t_{c}$ under oscillatory steady state for (a) small values of $j_{s}$ and (b) large values of $j_{s}$. Figure 2(a) shows that the evolution of $\psi_{s}(t)$ for small current densities $j_{s}$ featured a characteristic "kink" after the charging/discharging transitions. Similar kinks have also been 

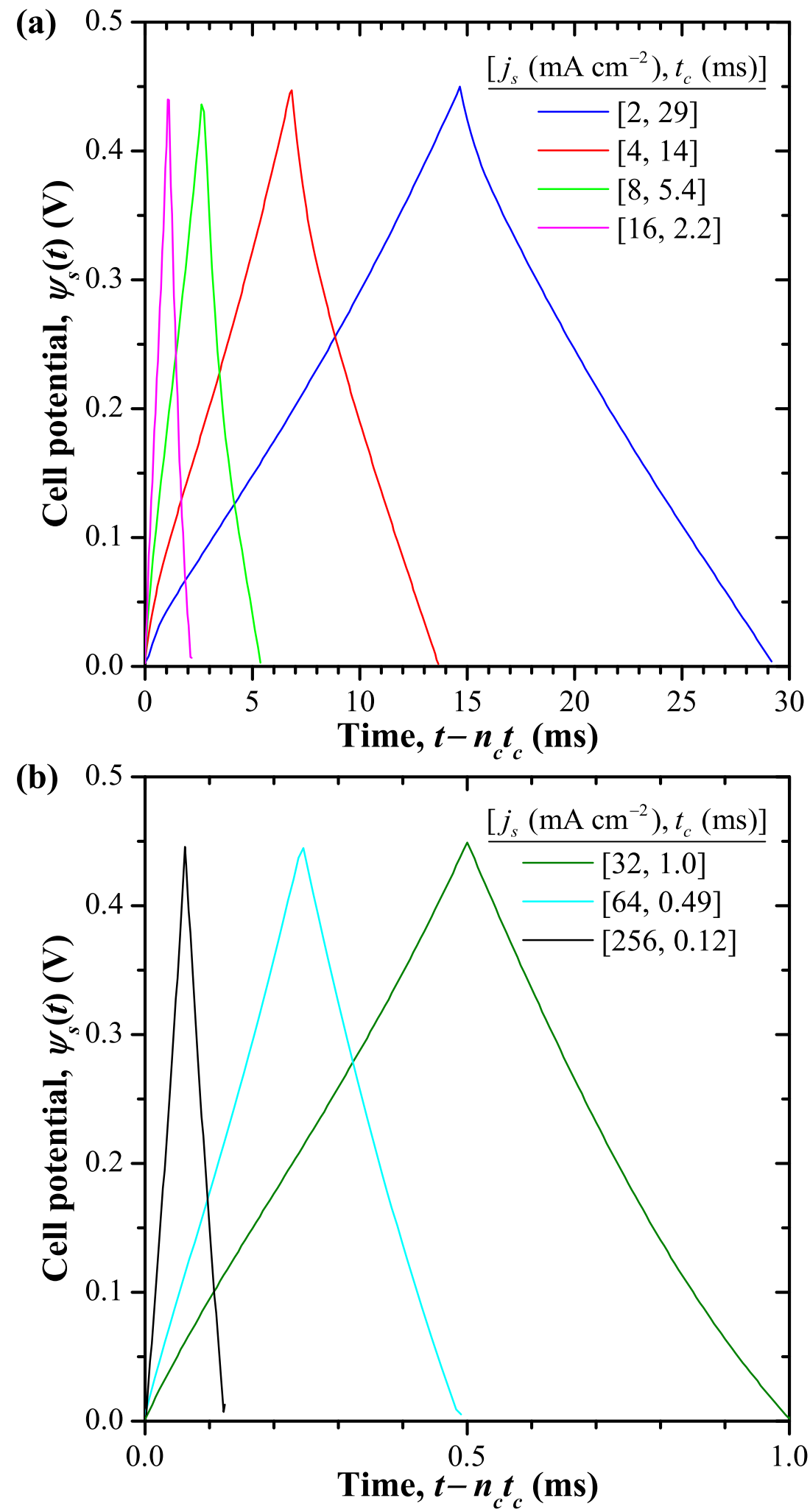

Figure 2: Predicted cell potential $\psi_{s}(t)$ as a function of time $t-n_{c} t_{c}$ for different current densities $j_{s}$ at oscillatory steady state between $\psi_{\min }=0 \mathrm{~V}$ and $\psi_{\max }=0.45 \mathrm{~V}$. 
observed experimentally for hybrid pseudocapacitors under galvanostatic cycling [32, 53-55]. They were reproduced numerically in Ref. [25] and were associated with a transition between capacitive-dominated and faradaic-dominated current. The relatively large slope $\left|\mathrm{d} \psi_{s} / \mathrm{d} t\right|$ immediately following the switch in the current direction can be attributed to a brief period of dominant capacitive current. Then, $\left|\mathrm{d} \psi_{s} / \mathrm{d} t\right|$ abruptly decreased as the faradaic current became dominant, resulting in the kink [25]. At large values of $j_{s}$, this faradaic kink vanished and the evolution of $\psi_{s}(t)$ became approximately symmetric around the peak $\psi_{s}=\psi_{\max }$.

Figure 3 shows the (a) faradaic $j_{F}(t) / j_{s}$ and (b) capacitive $j_{C}(t) / j_{s}$ fractions of the imposed current density as functions of dimensionless time $t / t_{c}$ at oscillatory steady state. The cycle period $t_{c}$ was retrieved from Figure 2. For small current densities $j_{s}$, the cell operated in the faradaic regime, i.e., the faradaic current dominated so that $j_{F}(t) \approx \pm j_{s}$ [Figure 3(a)] while the capacitive current was small except for brief peaks following the charging/discharging transitions [Figure 3(b)]. For large current densities $j_{s}$, the cell operated in the capacitive regime, i.e., $j_{C}(t) \approx \pm j_{s}$ [Figure $\left.3(\mathrm{~b})\right]$ while the faradaic current was small [Figure 3(a)]. These regimes are the same as those previously defined and analyzed in Ref. [25] for a hybrid pseudocapacitor charged by $\mathrm{Li}^{+}$intercalation under constant charge instead of constant potential window.

Figure $3(\mathrm{c})$ shows the intercalated $\mathrm{Li}^{+}$concentration $c_{1, P}(-L, t)$ in the pseudocapacitive electrode as a function of dimensionless time $t / t_{c}$. In all cases, $c_{1, P}$ was uniform throughout the pseudocapacitive electrode due to its small thickness $L_{P}=5 \mathrm{~nm}$. Figure 3(c) indicates that, in the faradaic regime, $c_{1, P}$ decreased almost linearly throughout the charging step as $\mathrm{Li}^{+}$deintercalated and increased throughout the discharging step as $\mathrm{Li}^{+}$intercalated. In fact, for $j_{s}=2 \mathrm{~mA} \mathrm{~cm}^{-2}$, the evolution of $c_{1, P}$ showed excellent agreement with the faradaic limit $c_{1, P, F}$ predicted by Equation (6). In the capacitive regime, $c_{1, P}$ was approximately constant due to the small faradaic current $j_{F}$ and equal to $c_{1, P, C}=0.8 \mathrm{~mol} \mathrm{~L}^{-1}$. Note that no $\mathrm{Li}^{+}$ starvation occurred inside the electrode for any of the cases considered with $\Delta \psi_{s}=0.45 \mathrm{~V}$.

Figure $3(\mathrm{~d})$ shows the surface overpotential $\eta(t)$ as a function of dimensionless time $t / t_{c}$. As previously observed in Ref. [25], the magnitude $|\eta(t)|$ was almost constant in the faradaic 
(a)
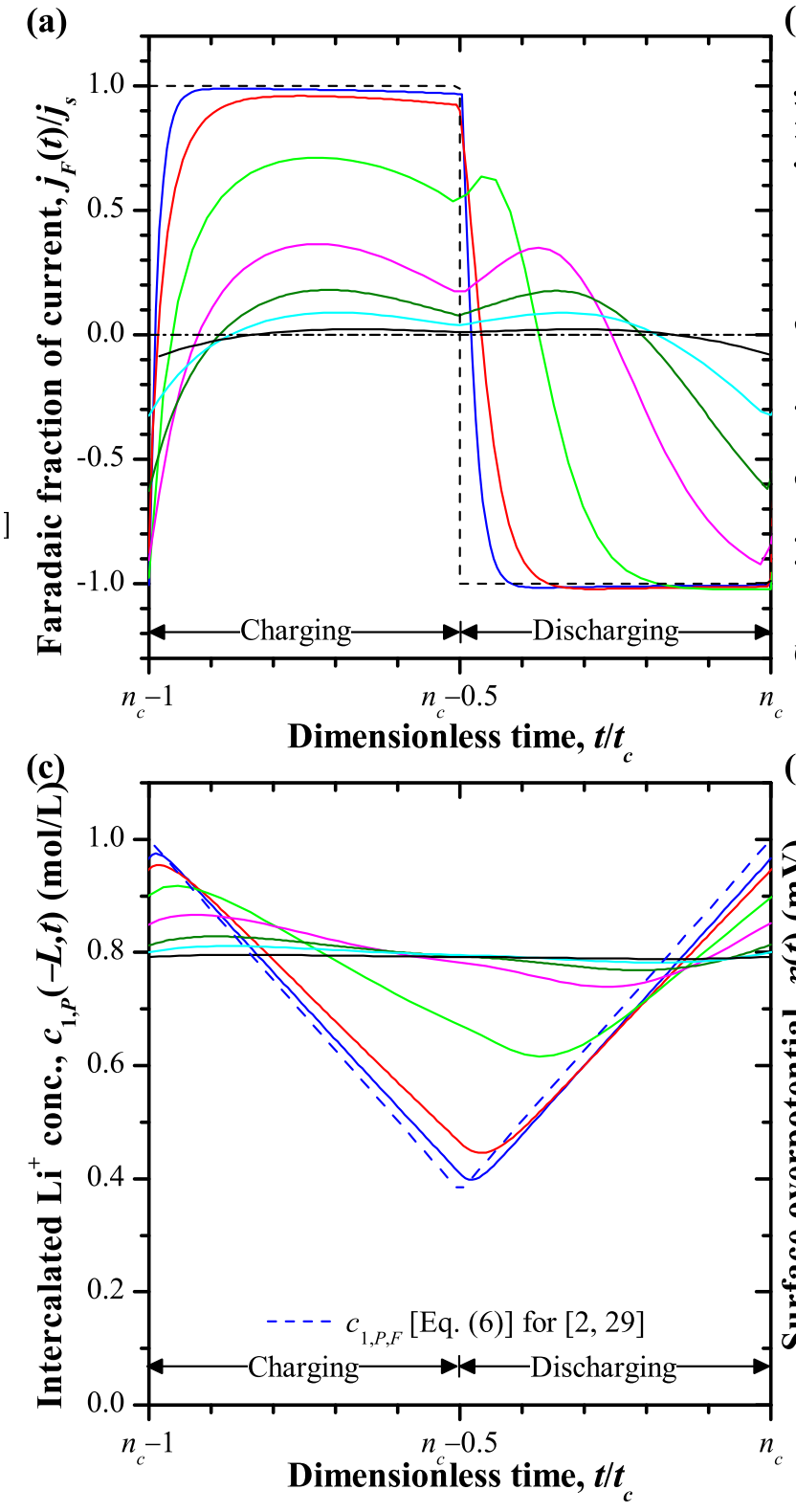

(b)

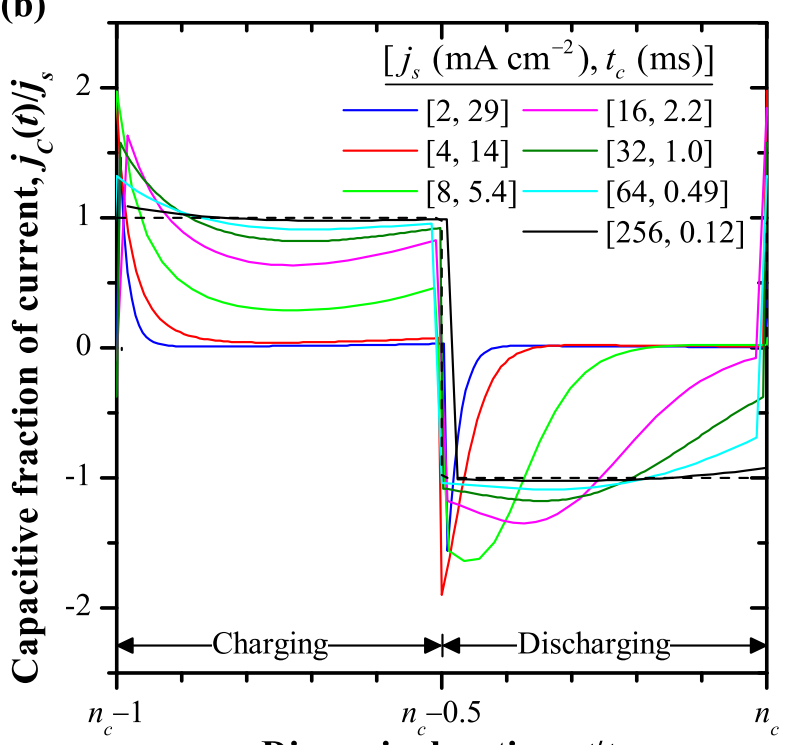

Dimensionless time, $t / t_{c}$

(d)

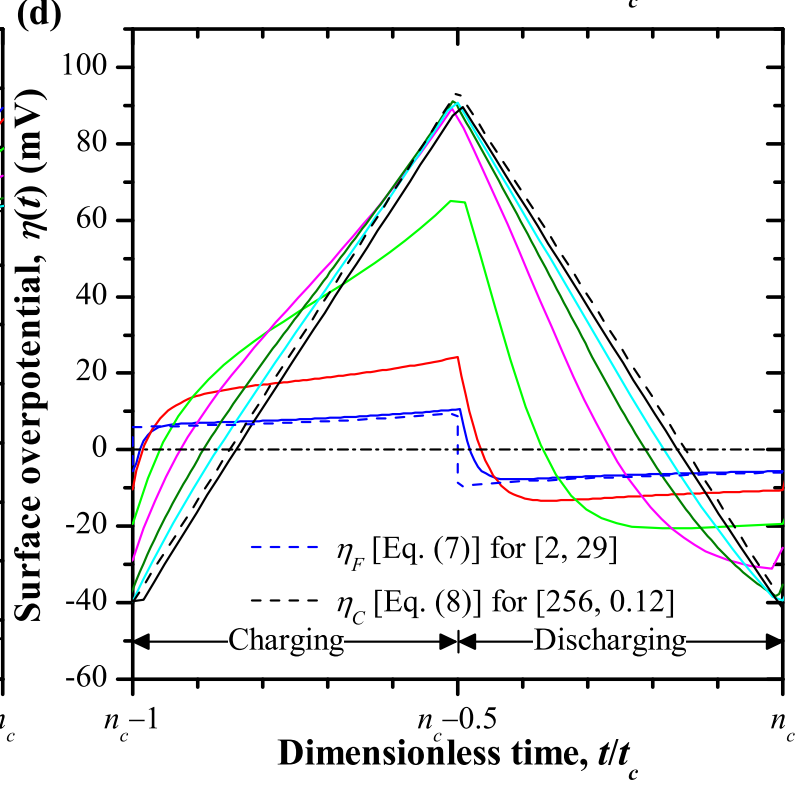

Figure 3: Predicted (a) faradaic $j_{F}(t) / j_{s}$ and (b) capacitive $j_{C}(t) / j_{s}$ fractions of the imposed current density as well as (c) intercalated $\mathrm{Li}^{+}$concentration $c_{1, P}(-L, t)$ and (d) surface overpotential $\eta(t)$ as functions of dimensionless time $t / t_{c}$. All cases are plotted over one cycle under oscillatory steady state for different values of $j_{s}$ and the same potential window $0 \mathrm{~V} \leq \psi_{s} \leq 0.45 \mathrm{~V}$. The analytical predictions (c) $c_{1, P, F}$ [Equation (6)] for $j_{s}=2 \mathrm{~mA} \mathrm{~cm}^{-2}$ in the faradaic regime as well as (d) $\eta_{F}$ [Equation (7)] for $j_{s}=2 \mathrm{~mA} \mathrm{~cm}{ }^{-2}$ in the faradaic regime and $\eta_{C}$ [Equation (8)] for $j_{s}=256 \mathrm{~mA} \mathrm{~cm}^{-2}$ in the capacitive regime are also shown. 
regime, except for brief transition periods immediately following the charging/discharging transitions. The evolution of $\eta(t)$ in the limits of small and large current density $j_{s}$ agreed well with analytical predictions $\eta_{F}(t)$ in the faradaic regime [Equation (7)] and $\eta_{C}(t)$ in the capacitive regime [Equation (8)].

Figure 4 shows the predicted (a) $\mathrm{Li}^{+}$concentration $c_{1, E}(-L+H, t)$ and (b) $\mathrm{ClO}_{4}^{-}$concentration $c_{2, E}(-L+H, t)$ at the Stern/diffuse layer interface near the pseudocapacitive electrode. In the faradaic regime, Figure 4 indicates that a $\mathrm{ClO}_{4}^{-}$EDL existed at the pseudocapacitive electrode during the charging step and was replaced by a $\mathrm{Li}^{+} \mathrm{EDL}$ during the discharging step. For large values of $j_{s}, \mathrm{a} \mathrm{Li}^{+}$EDL formed at the beginning and end of the cycle while a $\mathrm{ClO}_{4}^{-}$EDL formed at other times. The concentrations $c_{1, E}(-L+H, t)$ and $c_{2, E}(-L+H, t)$ were strongly correlated with the Stern layer electric field $E(-L+H, t)=$ $\Delta \psi_{H}(t) / H=\eta(t) / H$, with $\eta(t)$ reported in Figure 3(d). In the middle of the cycle, when the surface overpotential $\eta(t)$ and thus $E(-L+H, t)$ were positive and large [Figure 3(d)], the $\mathrm{Li}^{+}$ions were repelled and their concentration $c_{1, E}(-L+H, t)$ was small, approaching zero for $j_{s} \geq 8 \mathrm{~mA} \mathrm{~cm}^{-2}$. This $\mathrm{Li}^{+}$starvation decreased the faradaic current density $j_{F}(t)$, explaining the dip in $j_{F}$ around the charging/discharging transition at $t / t_{c}=n_{c}-0.5$ [Figure $3(\mathrm{a})]$. The positive peak in $j_{F}$ during the discharging step resulted from $c_{1, E}(-L+H, t)$ increasing as the $\mathrm{ClO}_{4}^{-}$EDL dissipated while $\eta(t)$ was still positive.

Figure 4 also shows the concentrations (c) $c_{1, E}(L-H, t)$ of $\mathrm{Li}^{+}$and $(\mathrm{d}) c_{2, E}(L-H, t)$ of $\mathrm{ClO}_{4}^{-}$near the carbon electrode. $\mathrm{A} \mathrm{Li}^{+}$EDL existed near the carbon electrode for the entire cycle without ever completely dissipating. This can be attributed to the fact that $\mathrm{Li}^{+}$originally from the pseudocapacitive electrode remained in the electrolyte, i.e., $c_{1, P}$ was smaller than its initial value $c_{1, P, 0}$ [Figure 3(c)]. As a result, the electrolyte retained a net positive charge even when the cell potential $\psi_{s}$ was zero, while the electrodes retained a corresponding negative charge.

\subsection{Heat generation rates}

In all cases, the Joule heat generation rate $\dot{q}_{J, i r r}$ in the diffuse layer of the electrolyte was found to be constant and uniform for galvanostatic cycling, except very close to the 
$\underline{\text { Near pseudocapacitive electrode }}$

(a)
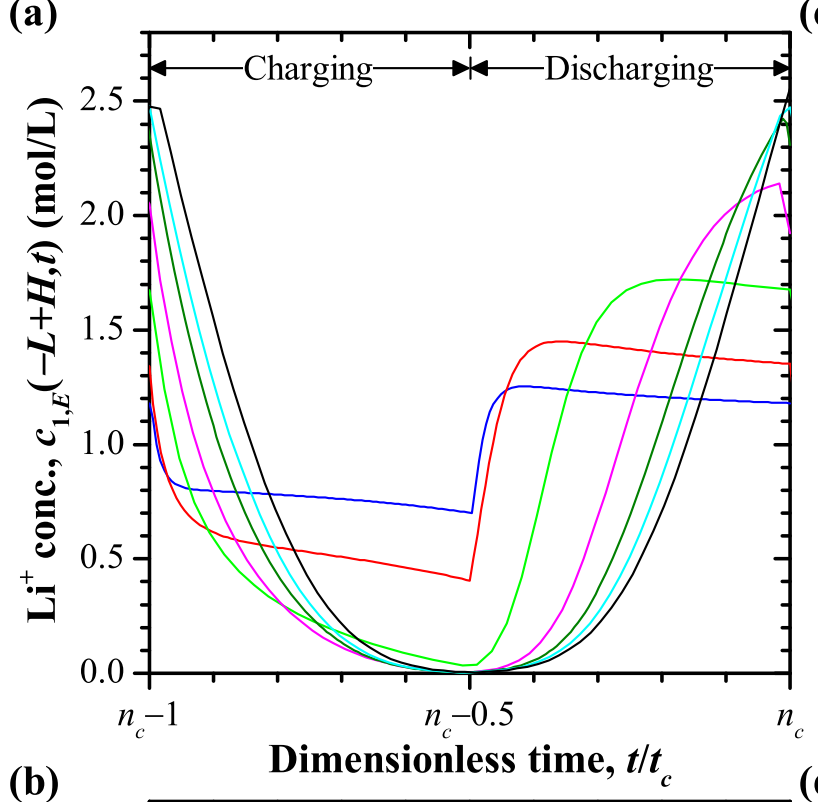

(b)

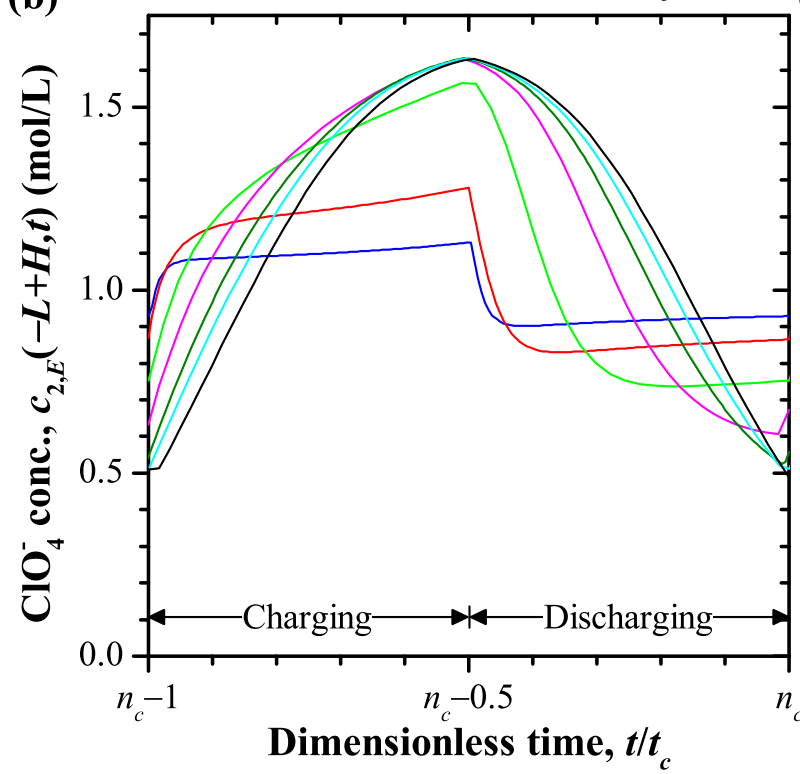

Near carbon electrode

(c)
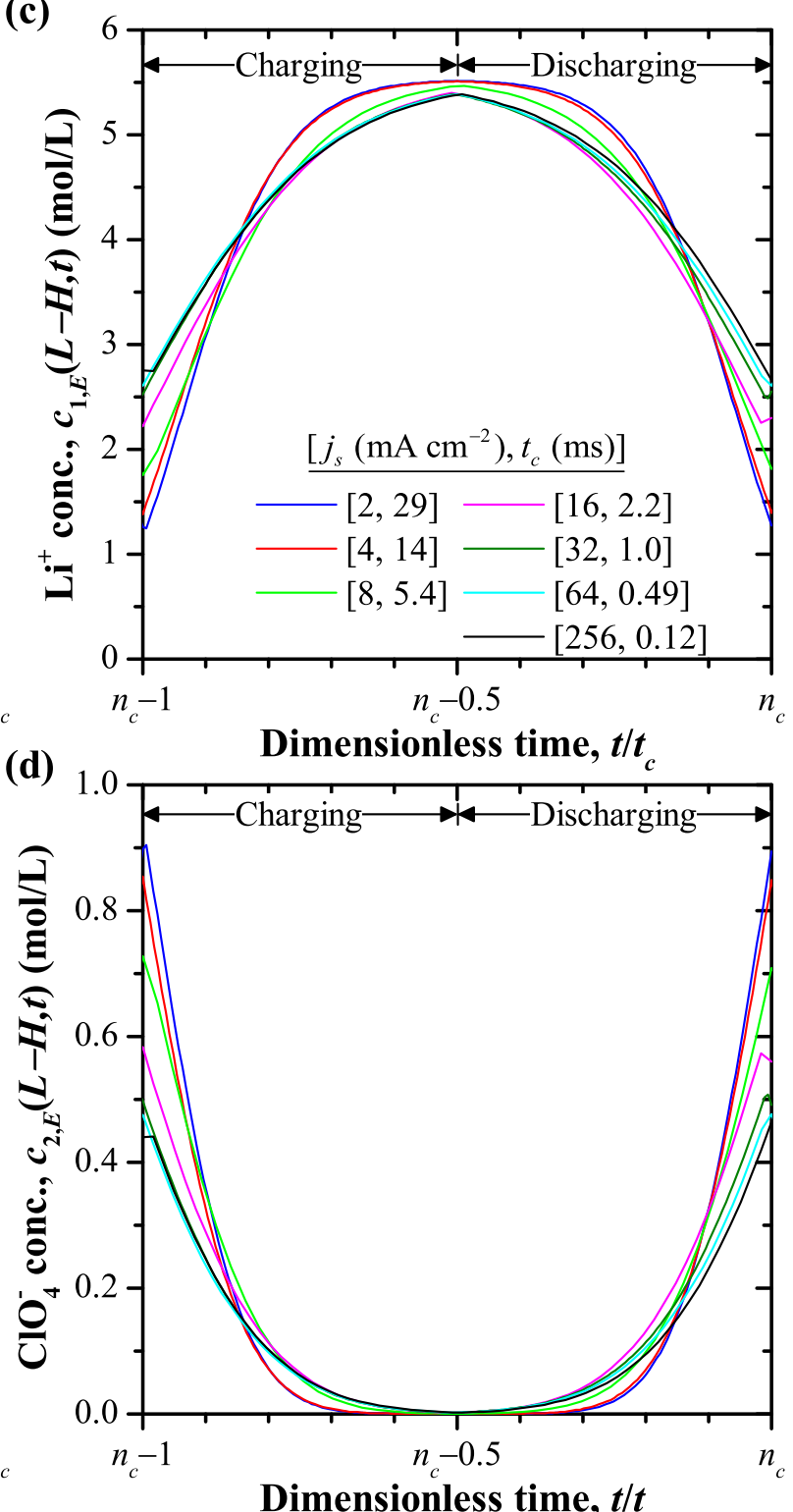

Figure 4: Predicted concentrations (a) $c_{1, E}(-L+H, t)$ of $\mathrm{Li}^{+}$and (b) $c_{2, E}(-L+H, t)$ of $\mathrm{ClO}_{4}^{-}$in the electrolyte near the pseudocapacitive electrode as well as (c) $c_{1, E}(L-H, t)$ of $\mathrm{Li}^{+}$and (d) $c_{2, E}(L-H, t)$ of $\mathrm{ClO}_{4}^{-}$near the carbon electrode. 
Stern/diffuse layer interfaces where it decreased sharply to zero, as observed in previous simulations of EDLCs $[11,12]$. It was also constant and uniform within each electrode. The total Joule heat generation rate $\dot{Q}_{J, i r r}^{\prime \prime}$ ranged from $3.9 \times 10^{-5}$ to $0.64 \mathrm{~mW} \mathrm{~cm}^{-2}$ as $j_{s}$ ranged from 2 to $256 \mathrm{~mA} \mathrm{~cm}^{-2}$.

\subsubsection{Heat generation rates in the pseudocapacitive electrode half-cell}

Figure 5 shows the total (a) irreversible faradaic $\dot{Q}_{F, i r r, P}^{\prime \prime}(t)$ and (b) reversible faradaic $\dot{Q}_{F, r e v, P}^{\prime \prime}(t)$ heat generation rates as well as the reversible EDL heat generation rate $\dot{Q}_{E D L, \text { rev }, P}^{\prime \prime}(t)$ of the pseudocapacitive electrode half-cell for (c) small and (d) large values of $j_{s}$ as functions of dimensionless time $t / t_{c}$. First, Figure $5(\mathrm{a})$ shows that the irreversible faradaic heat generation rate $\dot{Q}_{F, i r r, P}^{\prime \prime}$ was always positive. In the faradaic regime $\left(j_{s} \leq 4 \mathrm{~mA} \mathrm{~cm}{ }^{-2}\right)$, $\dot{Q}_{F, \text { irr }, P}^{\prime \prime}$ was proportional to the surface overpotential $\left|\eta_{F}(t)\right|$ given by Equation (7) such that $\dot{Q}_{F, i r r, P}^{\prime \prime} \approx j_{s}\left|\eta_{F}(t)\right|$ (see inset). The heat generation rate $\dot{Q}_{F, \text { irr }, P}^{\prime \prime}$ reached a maximum halfway through the cycle corresponding to the smallest $\mathrm{Li}^{+}$concentrations $c_{1, P}(-L, t)$ in the pseudocapacitive electrode and to the largest values of $\left|\eta_{F}(t)\right|$ [Figure 3(c)]. As $j_{s}$ increased, the maximum $\dot{Q}_{F, i r r, P}^{\prime \prime}$ increased. In addition, several distinct peaks in $\dot{Q}_{F, i r r, P}^{\prime \prime}$ were evident for large current densities $j_{s}$ in the capacitive regime. These peaks corresponded to those observed in the faradaic current density magnitude $\left|j_{F}(t)\right|$ [Figure 3(a)].

Figure 5(b) shows that the reversible faradaic heat generation rate $\dot{Q}_{F, r e v, P}^{\prime \prime}(t)$ was significantly larger in magnitude than its irreversible counterpart $\dot{Q}_{F, i r r, P}^{\prime \prime}(t)$ [Figure 5(a)] for all $j_{s}$ considered. It was also larger than the reversible EDL heat generation rate $\dot{Q}_{E D L, r e v, P}^{\prime \prime}(t)$ [Figure $5(\mathrm{c})$ ] for small $j_{s}$. This can be attributed to the fact that the surface overpotential $\eta(t)$ [Figure $3(\mathrm{~d})$ ] was smaller in magnitude $(|\eta(t)| \lesssim 0.1 \mathrm{~V})$ than the Peltier coefficient $|\Pi|=0.363 \mathrm{~V}$ for all $j_{s}$ considered and that the EDL contribution was small in the faradaic regime. Indeed, $\dot{Q}_{F, r e v, P}^{\prime \prime}(t)$ dominated $\dot{Q}_{P}^{\prime \prime}(t)$ [Equation (48)] for $j_{s} \leq 8 \mathrm{~mA} \mathrm{~cm}^{-2}$. In all cases, $\dot{Q}_{F, r e v, P}^{\prime \prime}(t)$ was directly proportional to $j_{F}(t)$ due to the constant value of $\Pi$ [Equations (32) and (46)]. Because $\Pi$ was negative, the sign of $\dot{Q}_{F, r e v, P}^{\prime \prime}$ was opposite to that of $j_{F}$. In other words, it was endothermic (i.e., negative) during charging by $\mathrm{Li}^{+}$deintercalation and exothermic (i.e., positive) during discharging by $\mathrm{Li}^{+}$intercalation. Thus, the mid-cycle 

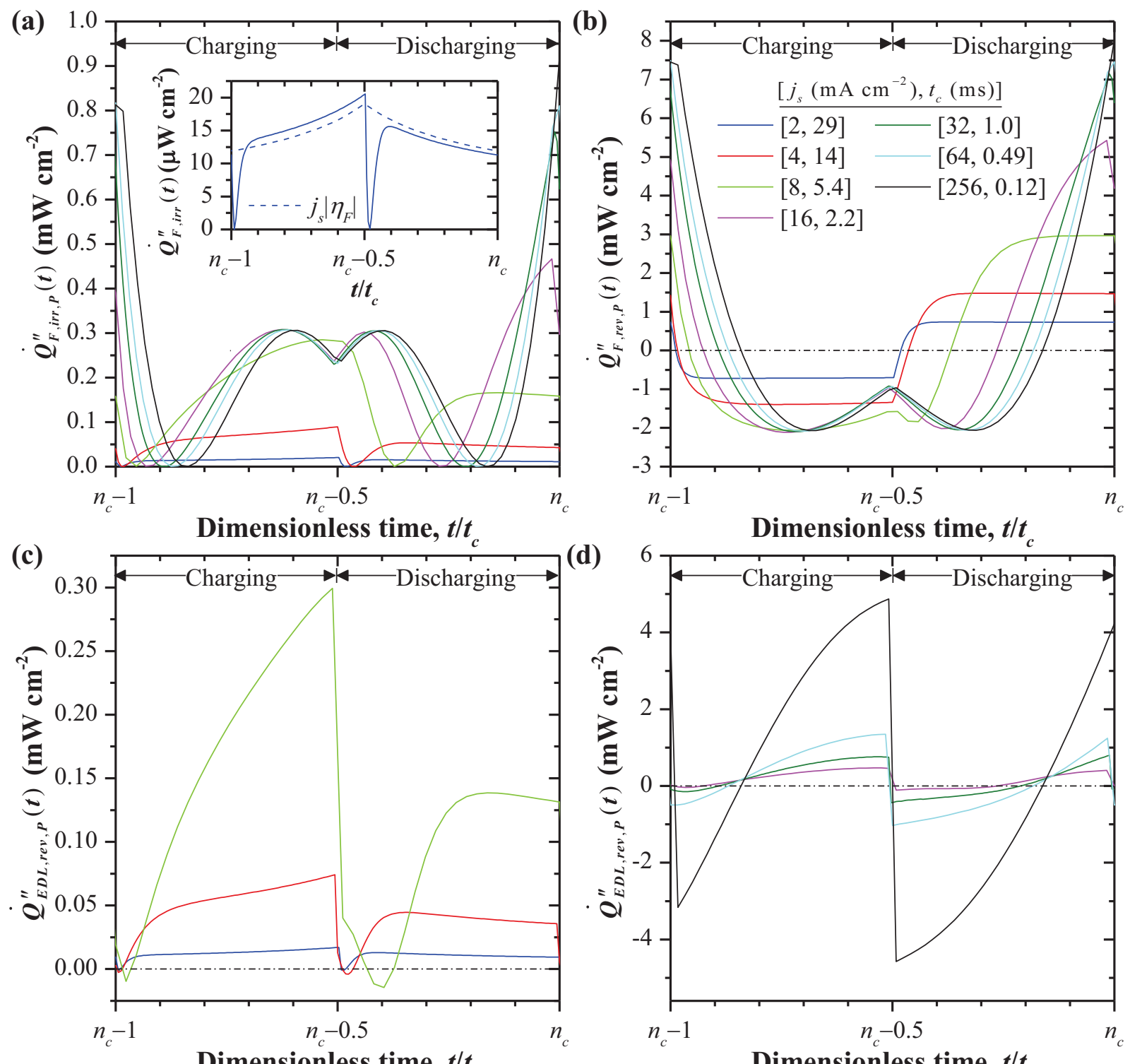

Figure 5: Predicted overall (a) irreversible faradaic $\dot{Q}_{F, i r r, P}^{\prime \prime}(t)$, (b) reversible faradaic $\dot{Q}_{F, \text { rev }, P}^{\prime \prime}(t)$, and reversible EDL $\dot{Q}_{E D L, r e v, P}^{\prime \prime}(t)$ heat generation rates for (c) small and (d) large current densities $j_{s}$ as functions of dimensionless time $t / t_{c}$. All cases are plotted over one cycle under oscillatory steady state for different values of $j_{s}$ and the same potential window $0 \mathrm{~V} \leq \psi_{s} \leq 0.45 \mathrm{~V}$. 
dip in $\left|\dot{Q}_{F, \text { rev }, P}^{\prime \prime}\right|$ and subsequent endothermic peak during discharging for $j_{s} \geq 8 \mathrm{~mA} \mathrm{~cm}-2$ can be attributed to $\mathrm{Li}^{+}$starvation limiting the faradaic current density $j_{F}$. Moreover, the time-averaged reversible faradaic heat generation $\overline{\dot{Q}}_{F, r e v, P}^{\prime \prime}$ over a cycle was zero at oscillatory steady state, since $\dot{Q}_{F, r e v, P}^{\prime \prime}(t)$ was proportional to $j_{F}(t)$ and no net $\mathrm{Li}^{+}$intercalation occurred, i.e., $\int_{\left(n_{c}-1\right) t_{c}}^{t_{c}} j_{F}(t) d t=0$.

Figures $5(\mathrm{c})$ and $(\mathrm{d})$ show that the magnitude of $\dot{Q}_{E D L, r e v, P}^{\prime \prime}(t)$ due to EDL formation increased with increasing $j_{s}$. However, the temporal evolution of $\dot{Q}_{E D L, \text { rev }, P}^{\prime \prime}$ near the pseudocapacitive electrode was distinctly different from that of $\dot{Q}_{E D L, r e v, C}^{\prime \prime}$ near the carbon electrode or in previous simulations of EDLCs $[11,12]$. For small current densities $j_{s}$ [Figure 5(c)], $\dot{Q}_{E D L, r e v, P}^{\prime \prime}(t)$ was exothermic during both the charging and the discharging steps. It also featured both exothermic and endothermic periods during each step for larger values of $j_{s}$ [Figure $5(\mathrm{~d})]$. Over a full cycle, the time average $\overline{\dot{Q}}_{E D L, r e v, P}^{\prime \prime}$ was positive for all $j_{s}$ considered. By contrast, the EDL reversible heating in EDLCs or in the carbon electrode half-cell was exothermic throughout the entire charging step, endothermic throughout the entire discharging step, and its time average $\bar{Q}_{E D L, \text { rev }, C}^{\prime \prime}$ over a full cycle was zero $[11,12]$. Indeed, near the carbon electrode at oscillatory steady state, the temporal evolution of the ion concentrations was symmetric around the charging/discharging transition [Figures 4(c) and 4(d)], while the direction of the current density changed sign. As a result, the reversible heat generation rates $\dot{q}_{E, d}, \dot{q}_{E, s}, \dot{q}_{S, c}$ [Equations (19) and (22)] in the carbon electrode half-cell were symmetric in magnitude but opposite in sign during charging and discharging. However, faradaic reactions at the pseudocapacitive electrode resulted in an asymmetic evolution of the ion concentrations [Figures $4(\mathrm{a})$ and $4(\mathrm{~b})$ ]. As a result, $\dot{Q}_{E D L, r e v, P}^{\prime \prime}(t)$ did not average to zero over the full cycle period. In other words, although the EDL heat generation rates are reversible, they behave reversibly only for symmetric EDL formation between charging and discharging such as that in EDLCs.

\subsubsection{Overall half-cell heat generation rates}

Figure 6 shows the numerically predicted overall heat generation rate (a) $\dot{Q}_{P}^{\prime \prime}(t)$ [Equation (48)] of the pseudocapacitive electrode half-cell and (b) $\dot{Q}_{C}^{\prime \prime}(t)$ [Equation (49)] of the carbon 


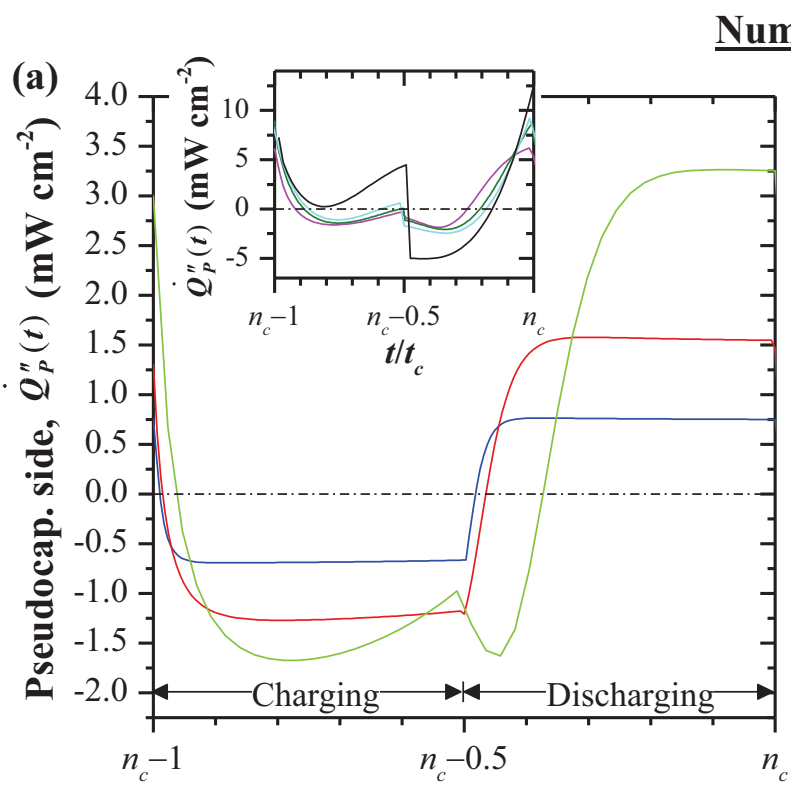

Dimensionless time, $t / t_{c}$

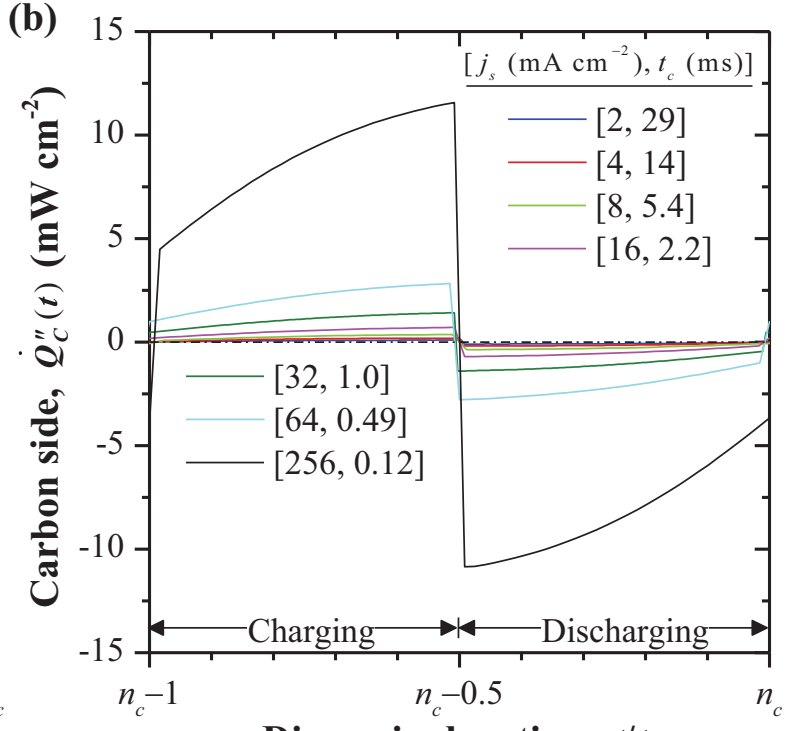

Dimensionless time, $t / t_{c}$

\section{$\underline{\text { Experimental }}$}

(c)

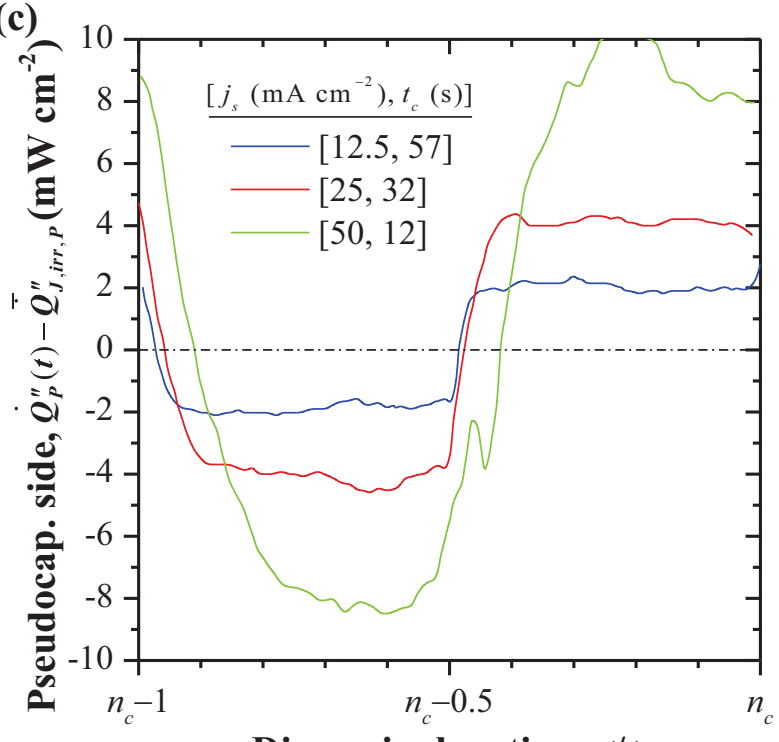

Dimensionless time, $t / t_{c}$

(d)

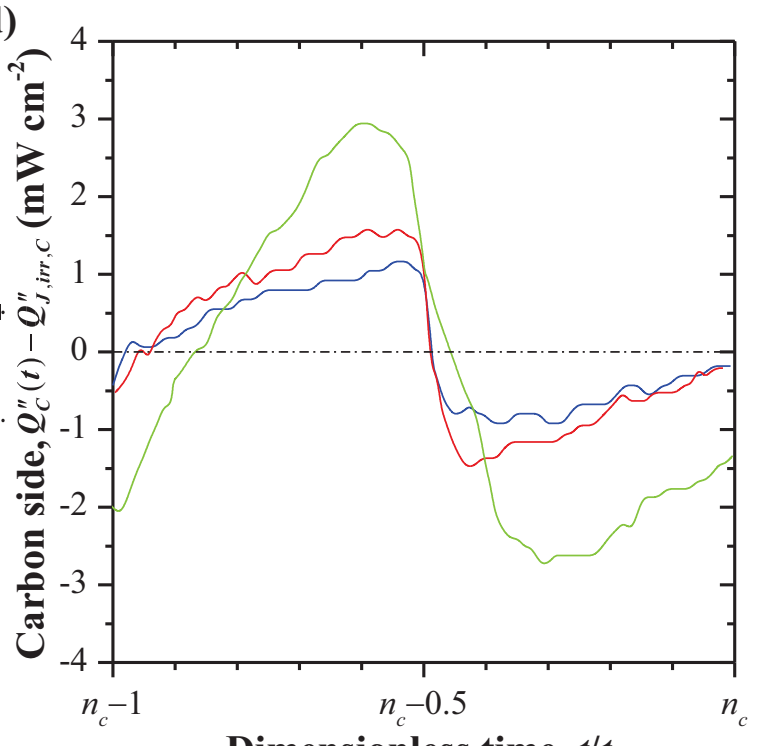

Figure 6: Numerically predicted total heat generation rates (a) $\dot{Q}_{P}^{\prime \prime}(t)$ of the pseudocapacitive electrode halfcell and (b) $\dot{Q}_{C}^{\prime \prime}(t)$ of the carbon electrode half-cell for $0 \mathrm{~V} \leq \psi_{s} \leq 0.45 \mathrm{~V}$ as well as experimentally measured total heat generation rates minus Joule heating $(\mathrm{c}) \dot{Q}_{P}^{\prime \prime}(t)-\bar{Q}_{J, i r r, P}^{\prime \prime}$ of the pseudocapacitive electrode half-cell and (d) $\dot{Q}_{C}^{\prime \prime}(t)-\bar{Q}_{J, i r r, C}^{\prime \prime}$ of the carbon electrode half-cell for a hybrid pseudocapacitor cycled galvanostatically over the potential window $0 \mathrm{~V} \leq \psi_{s} \leq 1.0 \mathrm{~V}[9]$ as functions of dimensionless time $t / t_{c}$ for different values of $j_{s}$. 
electrode half-cell as functions of dimensionless time $t / t_{c}$ over one cycle under oscillatory steady state for different values of $j_{s}$ and for $0 \mathrm{~V} \leq \psi_{s} \leq 0.45 \mathrm{~V}$. Figure 6(a) establishes that $\dot{Q}_{P}^{\prime \prime}(t)$ was endothermic during charging and exothermic during discharging for small current densities $j_{s}$, as the contribution $\dot{Q}_{F, r e v, P}^{\prime \prime}(t)$ dominated [Figure 5]. For larger values of $j_{s}, \dot{Q}_{P}^{\prime \prime}(t)$ featured both endothermic and exothermic periods during the charging and discharging steps. In particular, for $j_{s} \geq 8 \mathrm{~mA} \mathrm{~cm}^{-2}, \dot{Q}_{P}^{\prime \prime}(t)$ featured the endothermic peak during discharging previously noted in $\dot{Q}_{F, r e v, P}^{\prime \prime}$ and associated with $\mathrm{Li}^{+}$starvation. Figure $6(\mathrm{~b})$ indicates that $\dot{Q}_{C}^{\prime \prime}(t)$ was exothermic during charging and endothermic during discharging for all values of $j_{s}$ considered. This was consistent with the fact that the EDL always consisted of $\mathrm{Li}^{+}$[Figure 4(b)] and agreed with our previous EDLC simulations [11, 12].

Figure 6 also reproduces experimentally measured total heat generation rates minus constant Joule heating (c) $\dot{Q}_{P}^{\prime \prime}(t)-\overline{\dot{Q}}_{J, i r r, P}^{\prime \prime}$ for the porous $\mathrm{MnO}_{2}$ pseudocapacitive electrode half-cell and (d) $\dot{Q}_{C}^{\prime \prime}(t)-\overline{\dot{Q}}_{J, i r r, C}^{\prime \prime}$ for the carbon electrode half-cell as functions of dimensionless time $t / t_{c}$ for galvanostatic cycling over fixed potential window $0 \mathrm{~V} \leq \psi_{s} \leq 1.0 \mathrm{~V}$ (Figure 12 of Ref. [9]). Here, the authors subtracted the constant Joule heat generation rate estimated from the IR drops at charging/discharging transitions [9, 23]. The remainder was presented as the "reversible heat" associated with each electrode half-cell [9]. However, these values comprise all time-dependent contributions to the heat generation rate including the irreversible faradaic heat generation rate. For this reason, the experimental data are compared to the numerically predicted overall heat generation rates $\dot{Q}_{P}^{\prime \prime}(t)$ and $\dot{Q}_{C}^{\prime \prime}(t)$ rather than to the reversible contributions only. It is interesting to note that the experimental measurements for $j_{s}=12.5,25$, and $50 \mathrm{~mA} \mathrm{~cm}^{-2}$ qualitatively resembled the numerically predicted evolutions of $\dot{Q}_{P}^{\prime \prime}(t)$ and $\dot{Q}_{C}^{\prime \prime}(t)$ for $j_{s}=2,4$, and $8 \mathrm{~mA} \mathrm{~cm}^{-2}$ in both shape and in relative magnitudes for the different currents. For $j_{s}=50 \mathrm{~mA} \mathrm{~cm}{ }^{-2}$, experimentally $\dot{Q}_{P}^{\prime \prime}(t)-\overline{\dot{Q}}_{J, i r r, P}^{\prime \prime}[$ Figure $6(\mathrm{c})]$ featured an endothermic peak at the beginning of the discharging step very similar to that observed numerically in Figure 6 (a) for $j_{s}=8 \mathrm{~mA} \mathrm{~cm}^{-2}$ and caused by $\mathrm{Li}^{+}$starvation at the Stern/diffuse layer interface, as previously discussed. 


\subsubsection{Time-averaged heat generation rates}

Figure $7(\mathrm{a})$ shows the numerically predicted time-averaged heat generation rates over a full cycle at oscillatory steady state due to (i) Joule heating $\bar{Q}_{J, i r r}^{\prime \prime}$, (ii) irreversible faradaic heating $\overline{\dot{Q}}_{F, i r r, P}^{\prime \prime}$, and (iii) EDL reversible heating near the pseudocapacitive electrode $\overline{\dot{Q}}_{E D L, r e v, P}^{\prime \prime}$ as well as (iv) the total time-averaged heat generation rate for the entire cell $\bar{Q}^{\prime \prime}$ as functions of current density $j_{s}$ for $\psi_{\max }=0.45 \mathrm{~V}$ and for $\psi_{\max }=1.0 \mathrm{~V}$. First, as previously mentioned, $\bar{Q}_{F, r e v, P}^{\prime \prime}$ and $\bar{Q}_{E D L, r e v, C}^{\prime \prime}$ were zero and were not plotted. Thus, the total $\overline{\dot{Q}}^{\prime \prime}$ can be expressed as $\overline{\dot{Q}}^{\prime \prime}=\overline{\dot{Q}}_{J, i r r}^{\prime \prime}+\overline{\dot{Q}}_{F, i r r, P}^{\prime \prime}+\overline{\dot{Q}}_{E D L, r e v, P}^{\prime \prime}$. In addition, Figure $7($ a) indicates that $\overline{\dot{Q}}_{J, i r r}^{\prime \prime}$ was proportional to $j_{s}^{2}$ for all $j_{s}$, as indicated by Equation (44). It also establishes that, in the faradaic regime, $\bar{Q}_{F, i r r, P}^{\prime \prime}$ was proportional to $j_{s}^{2}$ for small values of $j_{s}$. This was consistent with the fact that, in this regime, $\left|j_{F}\right| \approx j_{s}$ and the magnitude of the surface overpotential $\eta_{F}$ was approximately proportional to $j_{s}$, as indicated by the first-order Taylor series approximation of Equation (7). Here, $\bar{Q}_{F, i r r, P}^{\prime \prime}$ was also several orders of magnitude larger than the Joule heat generation rate $\overline{\dot{Q}}_{J, i r r}^{\prime \prime}$ for small values of $j_{s}$ due to the small electrical resistance of the simulation domain. At larger current densities $j_{s}$, in the capacitive regime, $\bar{Q}_{F, i r r, P}^{\prime \prime}$ reached a plateau and became independent of $j_{s}$ because both $j_{F}$ and $\eta$ were found to be independent of $j_{s}[25]$. As a result, $\bar{Q}_{J, i r r}^{\prime \prime}$ eventually exceeded $\bar{Q}_{F, i r r, P}^{\prime \prime}$ for large enough values of $j_{s}$. Moreover, the average EDL heat generation rate $\overline{\dot{Q}}_{E D L, \text { rev }, P}^{\prime \prime}$ near the pseudocapacitive electrode was smaller but followed the same trend as $\bar{Q}_{F, i r r, P}^{\prime \prime}$ for all $j_{s}$. It provided a significant contribution to the net heat generated over a cycle, despite being a "reversible" heat generation rate. Finally, the overall average heat generation rate $\overline{\dot{Q}}$ "was (i) proportional to $j_{s}^{2}$ and dominated by $\overline{\dot{Q}}_{F, i r r, P}^{\prime \prime}$ and $\overline{\dot{Q}}_{E D L, r e v, P}^{\prime \prime}$ in the faradaic regime, (ii) proportional to $j_{s}^{2}$ and dominated by $\dot{Q}_{J, i r r}^{\prime \prime}$ in the capacitive regime, and (iii) featured a transition zone between these two regimes when it was independent of $j_{s}$. For the broader potential window $\Delta \psi_{s}=1.0 \mathrm{~V}$ and a given current $j_{s}$, the Joule heating $\overline{\dot{Q}}_{J, i r r}^{\prime \prime}$ remained identical to that for $\Delta \psi_{s}=0.45 \mathrm{~V}$. However, the irreversible faradaic $\bar{Q}_{F, \text { irr }, P}^{\prime \prime}$ and reversible EDL $\overline{\dot{Q}}_{E D L, r e v, P}^{\prime \prime}$ heat generation rates increased with increasing potential window. Indeed, for $\Delta \psi_{s}=1.0 \mathrm{~V}$, the intercalated $\mathrm{Li}^{+}$concentration $c_{1, P}$ reached smaller values than for 

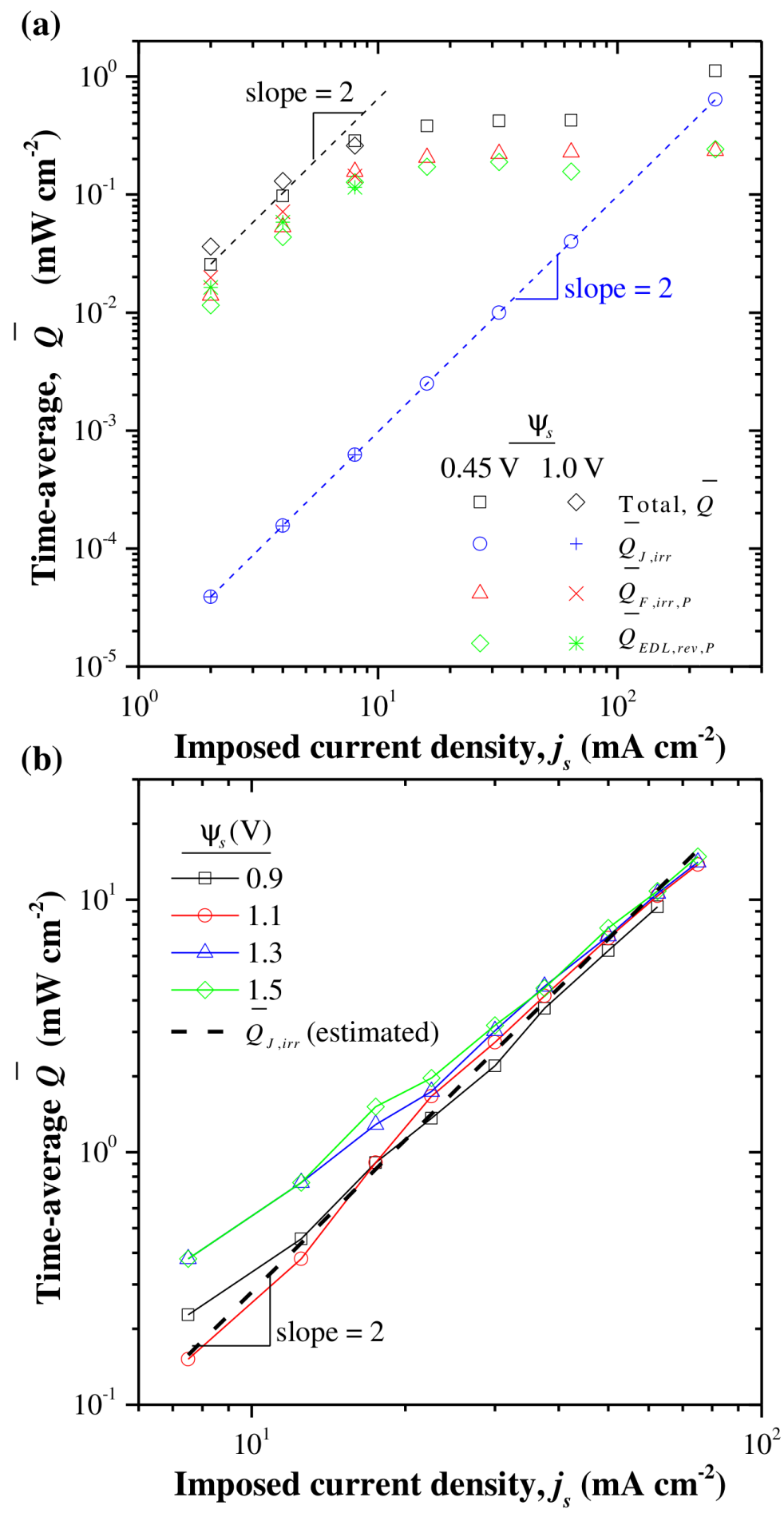

Figure 7: Time-averaged heat generation rates over a full cycle (a) predicted numerically due to Joule heating $\bar{Q}_{J, i r r}^{\prime \prime}$, irreversible faradaic heating $\bar{Q}_{F, i r r, P}^{\prime \prime}$, and EDL reversible heating near the pseudocapacitive electrode $\overline{\dot{Q}}_{E D L, \text { rev }, P}^{\prime \prime}$ as well as the overall $\overline{\dot{Q}}^{\prime \prime}=\overline{\dot{Q}}_{J, i r r}^{\prime \prime}+\overline{\dot{Q}}_{F, \text { irr }, P}^{\prime \prime}+\overline{\dot{Q}}_{E D L, \text { rev }, P}^{\prime \prime}$ and (b) overall $\overline{\dot{Q}}^{\prime \prime}$ measured experimentally [23] along with estimated Joule heating $\overline{\dot{Q}}_{J, i r r}^{\prime \prime}$ as functions of current density $j_{s}$. 
$\Delta \psi_{s}=0.45 \mathrm{~V}$, resulting in $\mathrm{Li}^{+}$starvation in the electrode for $j_{s}=2 \mathrm{~mA} \mathrm{~cm}^{-2}$ as well as longer periods of $\mathrm{Li}^{+}$starvation in the electrolyte. This resulted in larger values of $\eta(t)$ and larger EDLs near the pseudocapacitive electrode, thus increasing $\overline{\dot{Q}}_{F, i r r, P}^{\prime \prime}$ and $\overline{\dot{Q}}_{E D L, r e v, P}^{\prime \prime}$. As a result, the total heat generation rate $\bar{Q}^{\prime \prime}$ increased with increasing $\Delta \psi_{s}$ for small values of $j_{s}$.

Figure 7(b) reproduces experimentally measured time-averaged overall heat generation rates $\bar{Q}^{\prime \prime}$ reported by Dandeville [23] as a function of $j_{s}$ for several potential windows with $\psi_{\min }=0 \mathrm{~V}$ and $\psi_{\max }$ ranging from 0.9 and $1.5 \mathrm{~V}$ (Figure III.15 in Ref. [23]). The dashed line corresponds to estimated time-averaged Joule heating $\overline{\dot{Q}}_{J, i r r}^{\prime \prime}$ for the entire device. Here, we estimated the electrical resistance as $2.8 \Omega \mathrm{cm}^{2}$ based on the IR drop in $\psi_{s}(t)$ reported for $\Delta \psi_{s}=1.0 \mathrm{~V}$ and $j_{s}=25 \mathrm{~mA} \mathrm{~cm}^{-2}[9]$. For large values of $j_{s}, \overline{\dot{Q}}^{\prime \prime}$ fell approximately along the same line corresponding to the estimated Joule heat generation rate, for all potential windows. For small values of $j_{s}, \bar{Q}^{\prime \prime}$ exceeded the estimated $\bar{Q}_{J, i r r}^{\prime \prime}$, particularly for the broader potential windows $\Delta \psi_{s}=1.3 \mathrm{~V}$ and $1.5 \mathrm{~V}$. This behavior is qualitatively similar to that predicted numerically [Figure $7(\mathrm{a})]$ and attributed to $\overline{\dot{Q}}_{F, i r r, P}^{\prime \prime}$ and $\overline{\dot{Q}}_{E D L, \text { rev,P }}^{\prime \prime}$ increasing with increasing $\Delta \psi_{s}$ due to $\mathrm{Li}^{+}$starvation in the pseudocapacitive electrode and the electrolyte. The transition between the faradaic and capacitive regime behaviors was not as distinct experimentally, particularly for small $\Delta \psi_{s}$. This can be attributed to the relatively large electrical resistance and thus large experimental value of $\overline{\dot{Q}}_{J, i r r}^{\prime \prime}$.

\subsection{Temperature}

Figure 8 shows the predicted temperature $T(0, t)$ at the cell centerline as a function of time $t$ for $0 \mathrm{~V} \leq \psi_{s} \leq 0.45 \mathrm{~V}$ and (a) small and (b) large current densities $j_{s}$. For all current densities $j_{s}$, the temperature oscillated around an overall temperature rise $T_{i r r}(t)$ to irreversible heating. The slope of this irreversible temperature rise was proportional to the time-averaged heat generation rate $\dot{Q}^{\prime \prime}$ over a cycle and given by

$$
\frac{\mathrm{d} T_{i r r}}{\mathrm{~d} t}=\frac{\dot{Q}^{\prime \prime}}{C_{t h}}=\frac{\dot{\bar{Q}}_{J, i r r}^{\prime \prime}+\overline{\dot{Q}}_{F, i r r, P}^{\prime \prime}+\overline{\dot{Q}}_{E D L, r e v, P}^{\prime \prime}}{C_{t h}}
$$


(a)

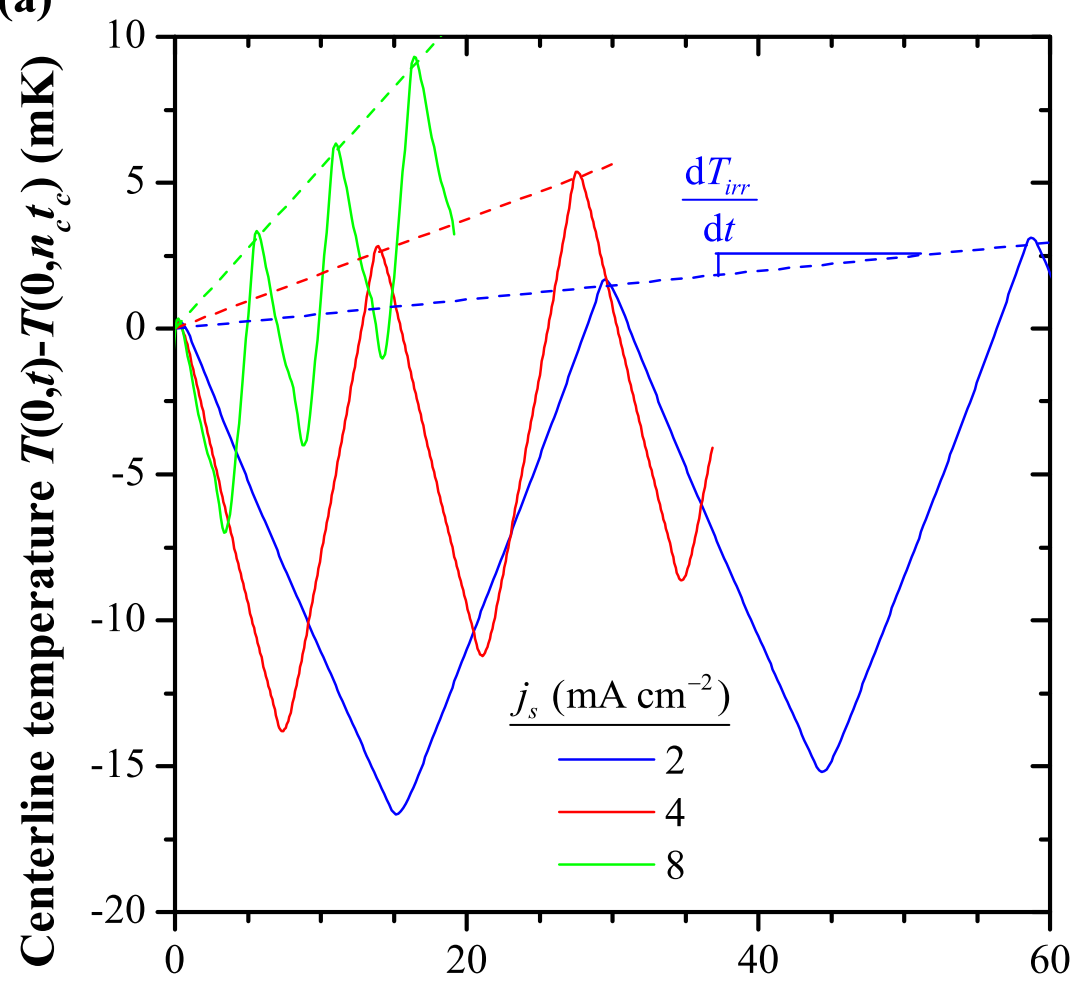

(b)
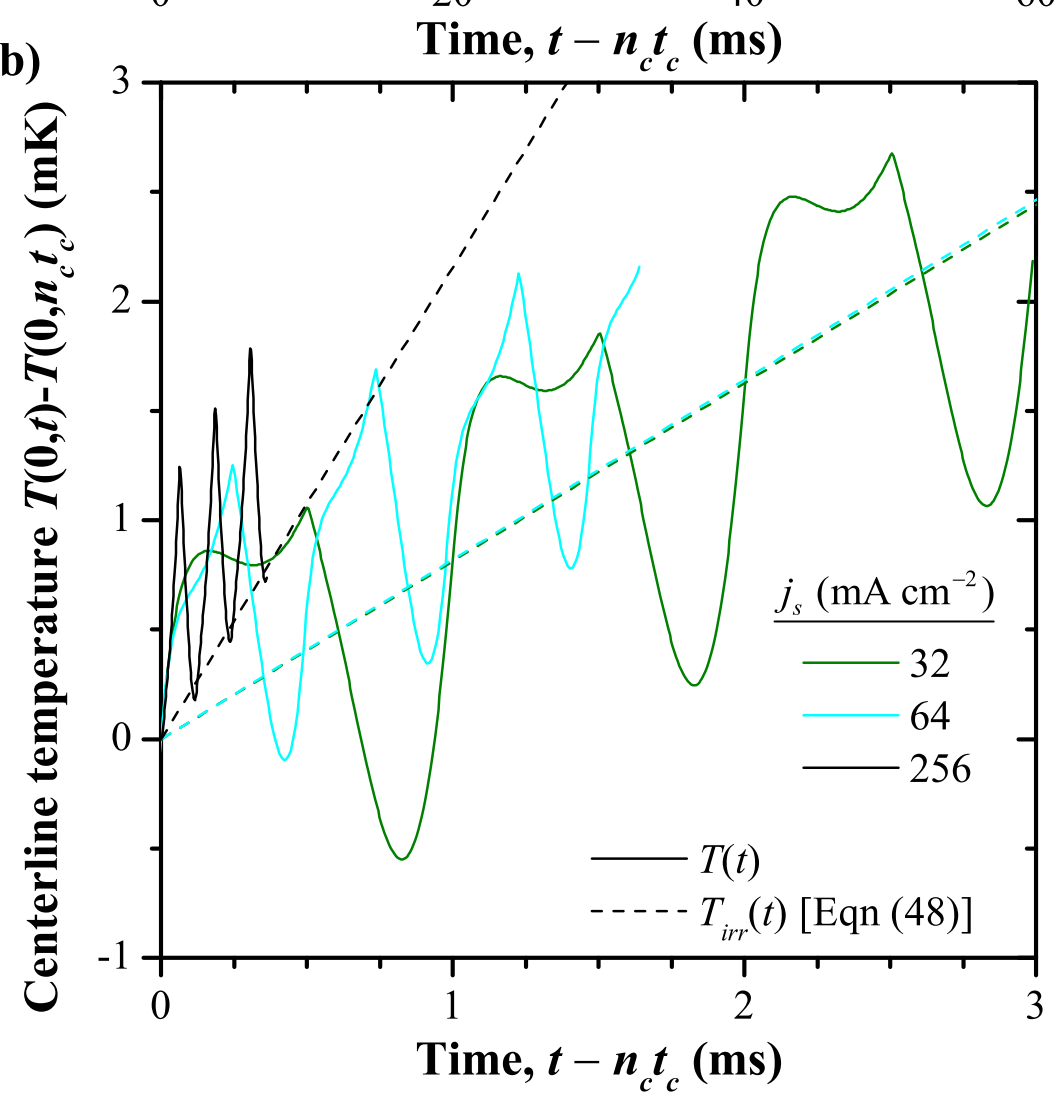

Figure 8: Predicted centerline temperature $T(0, t)-T\left(0, t_{c}\right)$ as a function of time $t$ over the potential window $0 \mathrm{~V} \leq \psi_{s} \leq 0.45 \mathrm{~V}$ for (a) small and (b) large current densities $j_{s}$. 
where $C_{t h}=\rho_{P} c_{p, P} L_{P}+2 \rho_{E} c_{p, E} L+\rho_{C} c_{p, C} L_{C}$ was the total heat capacity per unit surface area, equal to $5.18 \mathrm{~J} \mathrm{~m}^{-2} \mathrm{~K}^{-1}$.

Figure 8(a) shows that the slope $\mathrm{d} T_{\text {irr }} / \mathrm{d} t$ increased significantly with increasing $j_{s}$ as these

cases were in the faradaic regime when $\bar{Q}^{\prime \prime}$ was approximately proportional to $j_{s}^{2}$ [Figure $7(\mathrm{a})]$. The temperature oscillations had a triangular shape and the temperature initially decreased. This can be attributed to the dominance of $\dot{Q}_{F, r e v, P}^{\prime \prime}$, which was endothermic for $\mathrm{Li}^{+}$deintercalation, i.e., during the charging step, and approximately constant in magnitude [Figure 5(b)]. Similar triangular oscillations were measured experimentally for a hybrid pseudocapacitor charged by deintercalation and reported in Ref. [9].

By contrast, Figure $8(\mathrm{~b})$ shows that the slope $\mathrm{d} T_{\text {irr }} / \mathrm{d} t$ was approximately the same for $j_{s}=32$ and $64 \mathrm{~mA} \mathrm{~cm}^{-2}$. In fact, these cases were in the transition region observed in Figure $7\left(\right.$ a) when $\bar{Q}^{\prime \prime}$ was nearly independent of $j_{s}$. In addition, the temperature oscillations featured local maxima and minima within each cycle. This can be attributed to the fact that the total reversible heat generation rates due to the faradaic reaction $\dot{Q}_{F, \text { rev }, P}^{\prime \prime}$ and due to EDL formation $\dot{Q}_{E D L, \text { rev }, P}^{\prime \prime}$ and $\dot{Q}_{E D L, \text { rev }, C}^{\prime \prime}$ were of similar magnitude and had a significant effect on the temperature evolution. Finally, in the capacitive regime for $j_{s}=256 \mathrm{~mA} \mathrm{~cm}^{-2}$, the temperature oscillations were triangular and the temperature initially increased, similar to the EDLC temperatures reported in Ref. [9]. Here, $\dot{Q}_{C}^{\prime \prime}$ [Figure 6(b)] dominated heat generation.

\subsection{Coulombic efficiency}

Note that the electrochemical transport model summarized in Section 3.2 and the thermal model derived in the present study are idealized models with coulombic efficiency of $100 \%$, i.e., the chemical reaction described by Equation (1) occurs with no unwanted secondary reactions. Experimentally, the Coulombic efficiency is about 95-100\% [56]. Accounting for a coulombic efficiency of less than $100 \%$ would require the electrochemical transport model to be modified to include the relevant secondary reactions. Then, the faradaic current density $j_{F}(t)$ [Equation (3)] would have an individual contribution from each reaction occurring at the electrode. Additional mass conservation equations would arise for the concentrations 
of chemical species produced by the secondary reactions, and the ion and electron fluxes entering the Stern layers [Equation (28)] and boundary conditions] would depend on the stoichiometry and reaction rates of all reactions taking place. The local total volumetric heat generation rate $\dot{q}$ could then be evaluated in each region, accounting for all fluxes. However, such an analysis is beyond the scope of the present study.

\section{Conclusion}

The present study developed a first-principles continuum thermal model for hybrid pseudocapacitors accounting for local irreversible and reversible heat generation rates arising from Joule heating, faradaic reactions, and EDL formation. First, the temporal evolution of the different heat generation rates and of the temperature predicted numerically for a hybrid pseudocapacitor with planar electrodes charged by deintercalation of $\mathrm{Li}^{+}$under galvanostatic cycling showed excellent qualitative agreement with those measured experimentally [9]. This suggests that the model successfully captured the key physical phenomena governing the thermal behavior in pseudocapacitors. Interestingly, the predicted reversible heat generation rates due to EDL formation produced significant net heating over a cycle near the pseudocapacitive electrode and thus contributed to the overall temperature rise of the device. In addition, $\mathrm{Li}^{+}$starvation in the electrolyte resulted in a distinctive endothermic peak in the heat generation rate that was also observed experimentally. These observations indicate that both EDL formation and faradaic reactions must be considered simultaneously in thermal models of pseudocapacitors. Moreover, two asymptotic regimes of operation were observed, depending on the imposed cycling current density. In the faradaic regime, corresponding to small imposed current density and slow charging, irreversible faradaic and reversible EDL heating dominated the time-average heat generation rate. In the capacitive regime, corresponding to large imposed current density and fast charging, Joule heating dominated. The time-averaged heat generation rate $\bar{Q}^{\prime \prime}$ and the slope of the overall temperature rise were proportional to the square of the imposed current density $j_{s}^{2}$ in both the faradaic and capacitive regimes with different proportionality coefficients. In addition, $\bar{Q}^{\prime \prime}$ was nearly independent 
of $j_{s}$ in the transition region between the faradaic and capacitive regimes.

\section{Acknowledgement}

This material is based upon work supported by the National Science Foundation Graduate Research Fellowship under Grant No. DGE-1144087 and as part of the Molecularly Engineered Energy Materials, an Energy Frontier Research Center funded by the U.S. Department of Energy, Office of Science, Office of Basic Energy Sciences under Award Number DE-SC0001342.

\section{References}

[1] B. Conway, Electrochemical Supercapacitors: Scientific Fundamentals and Technological Applications, Kluwer Academic/Plenum Publishers, New York, NY, 1999.

[2] E. Frąckowiak, Electrode materials with pseudocapacitive properties, in: F. Béguin, E. Frąckowiak (Eds.), Supercapacitors: Materials, Systems, and Applications, WileyVCH Verlag, Weinheim, Germany, 2013, Ch. 6, pp. 207-237.

[3] C. A. Vincent, B. Scrosati (Eds.), Modern Batteries: An Introduction to Electrochemical Power Sources, 2nd Edition, Butterworth-Heinemann, Burlington, MA, 2003.

[4] T. Pandolfo, V. Ruiz, S. Sivakkumar, J. Nerkar, General properties of electrochemical capacitors, in: F. Béguin, E. Frąckowiak (Eds.), Supercapacitors: Materials, Systems, and Applications, Wiley-VCH Verlag, Weinheim, Germany, 2013, Ch. 2, pp. 69-109.

[5] US Department of Energy, Basic Research Needs for Electrical Energy Storage: Report of the Basic Energy Sciences Workshop for Electrical Energy Storage, Tech. rep., Office of Basic Energy Sciences, DOE (2007).

URL http://www.osti.gov/accomplishments/documents/fullText/ACC0330.pdf

[6] P. Simon, Y. Gogotsi, B. Dunn, Where do batteries end and supercapacitors begin?, Science 343 (2014) 1210-1211. 
[7] J. W. Kim, V. Augustyn, B. Dunn, The effect of crystallinity on the rapid pseudocapacitive response of $\mathrm{Nb}_{2} \mathrm{O}_{5}$, Advanced Energy Materials 2 (2012) 141-148.

[8] J. R. Miller, Electrochemical capacitor thermal management issues at high-rate cycling, Electrochimica Acta 52 (4) (2006) 1703-1708.

[9] Y. Dandeville, P. Guillemet, Y. Scudeller, O. Crosnier, L. Athouel, T. Brousse, Measuring time-dependent heat profiles of aqueous electrochemical capacitors under cycling, Thermochimica Acta 526 (2011) 1-8.

[10] J. Schiffer, D. Linzen, D. U. Sauer, Heat generation in double layer capacitors, Journal of Power Sources 160 (1) (2006) 765-772.

[11] A. L. d'Entremont, L. Pilon, First-principles thermal modeling of electric double layer capacitors under constant-current cycling, Journal of Power Sources 246 (2014) 887 898.

[12] A. L. d'Entremont, L. Pilon, Thermal effects of asymmetric electrolytes in electric double layer capacitors, Journal of Power Sources 273 (2015) 196-209.

[13] O. Bohlen, J. Kowal, D. U. Sauer, Ageing behaviour of electrochemical double layer capacitors: Part II. Lifetime simulation model for dynamic applications, Journal of Power Sources 173 (1) (2007) 626-632.

[14] P. Guillemet, Y. Scudeller, T. Brousse, Multi-level reduced-order thermal modeling of electrochemical capacitors, Journal of Power Sources 157 (1) (2006) 630-640.

[15] M. Al Sakka, H. Gualous, J. Van Mierlo, H. Culcu, Thermal modeling and heat management of supercapacitor modules for vehicle applications, Journal of Power Sources 194 (2) (2009) 581-587.

[16] H. Gualous, H. Louahlia-Gualous, R. Gallay, A. Miraoui, Supercapacitor thermal modeling and characterization in transient state for industrial applications, IEEE Transactions on Industry Applications 45 (3) (2009) 1035-1044. 
[17] A. Bard, L. Faulkner, Electrochemical Methods: Fundamentals and Applications, John Wiley \& Sons, New York, NY, 2001.

[18] V. Bagotsky, Fundamentals of Electrochemistry, John Wiley \& Sons, Hoboken, NJ, 2006.

[19] J. S. Newman, K. E. Thomas-Alyea, Electrochemical Systems, 3rd Edition, John Wiley \& Sons, Hoboken, NJ, 2004.

[20] K. Wang, L. Zhang, B. Ji, J. Yuan, The thermal analysis on the stackable supercapacitor, Energy 59 (2013) 440 - 444.

[21] C. Pascot, Y. Dandeville, Y. Scudeller, P. Guillemet, T. Brousse, Calorimetric measurement of the heat generated by a double-layer capacitor cell under cycling, Thermochimica Acta 510 (2010) 53-60.

[22] H. Gualous, H. Louahlia, R. Gallay, Supercapacitor characterization and thermal modelling with reversible and irreversible heat effect, IEEE Transactions on Power Electronics 26 (11) (2011) 3402-3409.

[23] Y. Dandeville, Analyse Thermique et Électrochimique de Supercondensateurs Carbone$\mathrm{MnO}_{2}$ en Milieu Aqueux, Ph.D. thesis, Université de Nantes (2012).

[24] S.-L. Kuo, N.-L. Wu, Investigation of pseudocapacitive charge-storage reaction of $\mathrm{MnO}_{2}$ - $\mathrm{nH}_{2} \mathrm{O}$ supercapacitors in aqueous electrolytes, Journal of The Electrochemical Society 153 (7) (2006) A1317-A1324.

[25] A. L. d'Entremont, H.-L. Girard, L. Pilon, Electrochemical transport phenomena in hybrid pseudocapacitors under galvanostatic cycling, Journal of The Electrochemical Society 163 (2) (2016) A229-A243.

[26] D. H. Lee, U. S. Kim, C. B. Shin, B. H. Lee, B. W. Kim, Y.-H. Kim, Modelling of the thermal behaviour of an ultracapacitor for a $42-\mathrm{V}$ automotive electrical system, Journal of Power Sources 175 (1) (2008) $664-668$. 
[27] J. Lee, J. Yi, D. Kim, C. B. Shin, K.-S. Min, J. Choi, H.-Y. Lee, Modeling of the electrical and thermal behaviors of an ultracapacitor, Energies 7 (2014) 8264-8278.

[28] A. L. d'Entremont, L. Pilon, First-order thermal model of commercial EDLCs, Applied Thermal Engineering 67 (2014) 439-446.

[29] C.-J. Chiang, J.-L. Yang, W.-C. Cheng, Temperature and state-of-charge estimation in ultracapacitors based on extended Kalman filter, Journal of Power Sources 234 (2013) $234-243$.

[30] V. Srinivasan, J. W. Weidner, Mathematical modeling of electrochemical capacitors, Journal of The Electrochemical Society 146 (1999) 1650-1658.

[31] M. S. Kilic, M. Z. Bazant, A. Ajdari, Steric effects in the dynamics of electrolytes at large applied voltages. II. Modified Poisson-Nernst-Planck equations, Physical Review E 75 (2) (2007) 021503.

[32] J. Come, V. Augustyn, J. W. Kim, P. Rozier, P.-L. Taberna, P. Gogotsi, J. W. Kong, B. Dunn, P. Simon, Electrochemical kinetics of nanostructured $\mathrm{Nb}_{2} \mathrm{O}_{5}$ electrodes, Journal of the Electrochemical Society 161 (2014) A718-A725.

[33] R. F. Probstein, Physicochemical Hydrodynamics: An Introduction, 2nd Edition, John Wiley \& Sons, Hoboken, NJ, 2003.

[34] R. B. Bird, W. E. Stewart, E. N. Lightfoot, Transport Phenomena, John Wiley \& Sons, New York, NY, 1962.

[35] H. Wang, A. Thiele, L. Pilon, Simulations of cyclic voltammetry for electric double layers in asymmetric electrolytes: A generalized modified Poisson-Nernst-Planck model, The Journal of Physical Chemistry C 117 (2013) 18286-18297.

[36] P. M. Biesheuvel, M. van Soestbergen, M. Z. Bazant, Imposed currents in galvanic cells, Electrochemica Acta 54 (21) (2009) 4857-4871. 
[37] A. Frumkin, Hydrogen overvoltage and the structure of the double layer, Zeitschrift für Elektrochemie 164A (1933) 121-133.

[38] C.-W. Wang, A. M. Sastry, Mesoscale modeling of a Li-ion polymer cell, Journal of the Electrochemical Society 154 (11) (2007) 1035-1047.

[39] S. R. Subramanian, V. Boovaragavan, V. Ramadesigan, M. Arabandi, Mathematical model reformulation for lithium-ion battery simulations: Galvanostatic boundary conditions, Journal of the Electrochemical Society 156 (4) (2009) 260-271.

[40] F. P. Incropera, D. P. DeWitt, T. L. Bergman, A. S. Lavine, Fundamentals of Heat and Mass Transfer, 6th Edition, John Wiley \& Sons, Hoboken, NJ, 2007.

[41] W. B. Gu, C. Y. Wang, Thermal-electrochemical modeling of battery systems, Journal of the Electrochemical Society 147 (8) (2000) 2910-2922.

[42] E. R. Cohen, T. Cvitas, J. G. Frey, B. Holmström, K. Kuchitsu, R. Marquardt, I. Mills, F. Pavese, M. Quack, J. Stohner, H. . Strauss, M. Takami, A. J. Thor (Eds.), Quantities, Units and Symbols in Physical Chemistry, 3rd Edition, IUPAC Green Book, IUPAC and RSC Publishing, Cambridge, UK, 2008.

URL http://www.iupac.org/fileadmin/user_upload/publications/ e-resources/ONLINE-IUPAC-GB3-2ndPrinting-Online-Sep2012.pdf

[43] M. A. Liberman, Introduction to Physics and Chemistry of Combustion, Springer-Verlag Berlin Heidelberg, 2008.

[44] P. Debye, E. Hückel, Zur Theorie der Elektrolyte. I. Gefrierpunktserniedrigung und verwandte Erscheinungen, Physikalische Zeitschrift 24 (1923) 185-206.

[45] T. Engel, P. Reid, Thermodynamics, Statistical Thermodynamics, and Kinetics, 2nd Edition, Prentice Hall, Upper Saddle River, NJ, 2010.

[46] J. E. Schlewitz, Niobium and niobium compounds, in: Kirk-Othmer Encyclopedia of Chemical Technology, John Wiley \& Sons, Inc., 2009, pp. 1-27. 
[47] E. S. Domalski, E. D. Hearing, "Condensed phase heat capacity data", in: P. J. Linstrom, W. G. Mallard (Eds.), NIST Chemistry WebBook, NIST Standard Reference Database Number 69, National Institute of Standards and Technology, Gaithersburg, MD, 2011.

[48] R. W. Powell, C. Y. Ho, P. E. Liley, Thermal conductivity of selected materials, National Standard Reference Data Series. (NSRDS-NBS 8), U.S. Dept. of Commerce, National Bureau of Standards, Washington, D.C., 1966.

[49] W. M. Haynes, T. J. Bruno, D. R. Lide (Eds.), CRC Handbook of Chemistry and Physics, 94th Edition, CRC Press, Boca Raton, FL, 2013.

[50] Design Institute for Physical Properties, DIPPR Project 801 - Full version, American Institute of Chemical Engineers (AIChE) (2012).

URL

http://app.knovel.com/hotlink/toc/id:kpDIPPRPF7/

dippr-project-801-full

[51] Q. Huang, M. Yan, Z. Jiang, Thermal study on single electrodes in lithium-ion battery, Journal of Power Sources 156 (2006) 541-546.

[52] COMSOL, Inc., COMSOL Multiphysics Reference Manual, COMSOL 4.4 Edition (2013).

[53] M. S. Hong, S. H. Lee, S. W. Kim, Use of $\mathrm{KCl}$ aqueous electrolyte for $2 \mathrm{~V}$ manganese oxide/activated carbon hybrid capacitor, Electrochemical and Solid-State Letters 5 (10) (2002) A227-A230.

[54] E. Lim, H. Kim, C. Jo, J. Chun, K. Ku, S. Kim, H. I. Lee, I.-S. Nam, S. Yoon, K. Kang, J. Lee, Advanced hybrid supercapacitor based on a mesoporous niobium pentoxide/carbon as high-performance anode, ACS Nano 8 (9) (2014) 8968-8978.

[55] L. Kong, C. Zhang, S. Zhang, J. Wang, R. Cai, C. Lv, W. Qiao, L. Ling, D. Long, Highpower and high-energy asymmetric supercapacitors based on $\mathrm{Li}^{+}$-intercalation into a $\mathrm{T}$ - 
$\mathrm{Nb}_{2} \mathrm{O}_{5}$ /graphene pseudocapacitive electrode, Journal of Materials Chemistry A 2 (42) (2014) 17962-17970.

[56] M. Stoller, R. Ruoff, Best practice methods for determining an electrode material's performance for ultracapacitors, Energy Environ. Sci. 3 (2010) 1294-1301. 


\section{List of Figures}

1 Illustration of the simulated hybrid pseudocapacitor with planar electrodes. The electrolyte was divided into the Stern and diffuse layers according to the Stern model $[17,18] . \ldots \ldots \ldots \ldots$

2 Predicted cell potential $\psi_{s}(t)$ as a function of time $t-n_{c} t_{c}$ for different current densities $j_{s}$ at oscillatory steady state between $\psi_{\min }=0 \mathrm{~V}$ and $\psi_{\max }=0.45 \mathrm{~V} .24$

3 Predicted (a) faradaic $j_{F}(t) / j_{s}$ and (b) capacitive $j_{C}(t) / j_{s}$ fractions of the imposed current density as well as (c) intercalated $\mathrm{Li}^{+}$concentration $c_{1, P}(-L, t)$ and (d) surface overpotential $\eta(t)$ as functions of dimensionless time $t / t_{c}$. All cases are plotted over one cycle under oscillatory steady state for different values of $j_{s}$ and the same potential window $0 \mathrm{~V} \leq \psi_{s} \leq 0.45 \mathrm{~V}$. The analytical predictions (c) $c_{1, P, F}$ [Equation (6)] for $j_{s}=2 \mathrm{~mA} \mathrm{~cm}^{-2}$ in the faradaic regime as well as (d) $\eta_{F}$ [Equation (7)] for $j_{s}=2 \mathrm{~mA} \mathrm{~cm}^{-2}$ in the faradaic regime and $\eta_{C}$ [Equation (8)] for $j_{s}=256 \mathrm{~mA} \mathrm{~cm}^{-2}$ in the capacitive regime are also

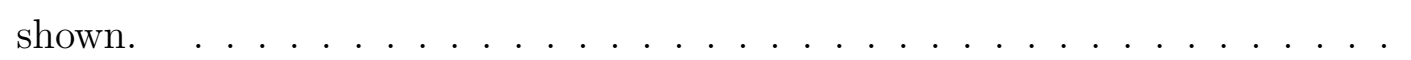

4 Predicted concentrations (a) $c_{1, E}(-L+H, t)$ of $\mathrm{Li}^{+}$and (b) $c_{2, E}(-L+H, t)$ of $\mathrm{ClO}_{4}^{-}$in the electrolyte near the pseudocapacitive electrode as well as (c) $c_{1, E}(L-H, t)$ of $\mathrm{Li}^{+}$and $(\mathrm{d}) c_{2, E}(L-H, t)$ of $\mathrm{ClO}_{4}^{-}$near the carbon electrode. 28

5 Predicted overall (a) irreversible faradaic $\dot{Q}_{F, i r r, P}^{\prime \prime}(t)$, (b) reversible faradaic $\dot{Q}_{F, r e v, P}^{\prime \prime}(t)$, and reversible EDL $\dot{Q}_{E D L, r e v, P}^{\prime \prime}(t)$ heat generation rates for (c) small and (d) large current densities $j_{s}$ as functions of dimensionless time $t / t_{c}$. All cases are plotted over one cycle under oscillatory steady state for different values of $j_{s}$ and the same potential window $0 \mathrm{~V} \leq \psi_{s} \leq 0.45 \mathrm{~V}$. . 
6 Numerically predicted total heat generation rates (a) $\dot{Q}_{P}^{\prime \prime}(t)$ of the pseudocapacitive electrode half-cell and (b) $\dot{Q}_{C}^{\prime \prime}(t)$ of the carbon electrode half-cell for $0 \mathrm{~V} \leq \psi_{s} \leq 0.45 \mathrm{~V}$ as well as experimentally measured total heat generation rates minus Joule heating (c) $\dot{Q}_{P}^{\prime \prime}(t)-\bar{Q}_{J, i r r, P}^{\prime \prime}$ of the pseudocapacitive electrode half-cell and (d) $\dot{Q}_{C}^{\prime \prime}(t)-\dot{Q}_{J, i r r, C}^{\prime \prime}$ of the carbon electrode half-cell for a hybrid pseudocapacitor cycled galvanostatically over the potential window $0 \mathrm{~V} \leq \psi_{s} \leq 1.0 \mathrm{~V}[9]$ as functions of dimensionless time $t / t_{c}$ for different values of $j_{s} \ldots \ldots \ldots \ldots \ldots \ldots$

7 Time-averaged heat generation rates over a full cycle (a) predicted numerically due to Joule heating $\overline{\dot{Q}}_{J, i r r}^{\prime \prime}$, irreversible faradaic heating $\overline{\dot{Q}}_{F, i r r, P}^{\prime \prime}$, and EDL reversible heating near the pseudocapacitive electrode $\overline{\dot{Q}}_{E D L, \text { rev,P }}^{\prime \prime}$ as well as the overall $\overline{\dot{Q}}^{\prime \prime}=\overline{\dot{Q}}_{J, i r r}^{\prime \prime}+\overline{\dot{Q}}_{F, i r r, P}^{\prime \prime}+\overline{\dot{Q}}_{E D L, r e v, P}^{\prime \prime}$ and (b) overall $\dot{\bar{Q}}^{\prime \prime}$ measured experimentally [23] along with estimated Joule heating $\bar{Q}_{J, i r r}^{\prime \prime}$ as functions of current density $j_{s} \ldots \ldots \ldots \ldots \ldots \ldots \ldots$

8 Predicted centerline temperature $T(0, t)-T\left(0, t_{c}\right)$ as a function of time $t$ over the potential window $0 \mathrm{~V} \leq \psi_{s} \leq 0.45 \mathrm{~V}$ for (a) small and (b) large current

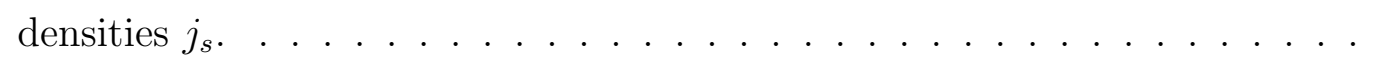

